\title{
Oxidative Stress Induces Neuronal Apoptosis Through Suppressing Transcription Factor EB Phosphorylation at Ser467
}

\author{
Qian Su $^{\mathrm{a}}$ Bin Zheng ${ }^{\mathrm{a}}$ Chen-yao Wang ${ }^{\mathrm{b}}$ Yun-zhi Yang ${ }^{\mathrm{b}}$ Wen-wen Luo \\ Shu-min Ma ${ }^{b}$ Xin-hua Zhang ${ }^{a}$ Dong Ma ${ }^{a}$ Yan Sun ${ }^{a}$ Zhan Yang ${ }^{a}$ Jin-kun Wen \\ Zhi-xue Liub

\begin{abstract}
aDepartment of Biochemistry and Molecular Biology, The Key Laboratory of Neural and Vascular Biology, Ministry of Education, Hebei Medical University, Shijiazhuang, Hebei, ${ }^{b}$ Key Laboratory of Nutrition and Metabolism, Institute for Nutritional Sciences, Shanghai Institutes for Biological Sciences, Chinese Academy of Sciences University of Chinese Academy of Sciences, Shanghai, China
\end{abstract}

\section{Key Words}

Tfeb • Akt signalling • Phosphorylation • Oxidative stress • SH-SY5Y cell • Neuronal apoptosis

\begin{abstract}
Background/Aims: This study determined the role and mechanism of action of transcription factor EB (TFEB) in $\mathrm{H}_{2} \mathrm{O}_{2}$-induced neuronal apoptosis. Methods: $\mathrm{SH}-\mathrm{SY} 5 \mathrm{Y}$ cells were treated with Akt inhibitor/activator and different concentrations of $\mathrm{H}_{2} \mathrm{O}_{2}$. Cell apoptosis was detected by flow cytometric analysis. Akt and TFEB phosphorylation and PARP cleavage were determined by Western blotting. HEK293T cells were transfected with different truncated TFEB mutants and HA-Akt-WT; SH-SY5Y cells were transfected with Flag-vector, Flag-TFEB, Flag-TFEB-S467A or Flag-TFEB-S467D; and TFEB interaction with Akt was determined by coimmunoprecipitation and GST pull-down assays. Results: A low concentration of $\mathrm{H}_{2} \mathrm{O}_{2}$ induces TFEB phosphorylation at Ser467 and nuclear translocation, facilitating neuronal survival, whereas a high concentration of $\mathrm{H}_{2} \mathrm{O}_{2}$ promotes $\mathrm{SH}-\mathrm{SY} 5 Y$ cell apoptosis via suppressing TFEB Ser467 phosphorylation and nuclear translocation. The TFEB-S467D mutant is more easily translocated into the nucleus than the non-phosphorylated TFEB-S467A mutant. Further, Akt physically binds to TFEB via its C-terminal tail interaction with the HLH domain of TFEB and phosphorylates TFEB at Ser467. Mutation of TFEB-Ser467 can prevent the phosphorylation of TFEB by Akt, preventing inhibition of oxidative stress-induced apoptosis. Conclusions: Oxidative stress induces neuronal apoptosis through suppressing TFEB phosphorylation at Ser467 by Akt, providing a novel therapeutic strategy for neurodegenerative diseases.
\end{abstract}

\section{KARGER}




\section{Cellular Physiology Cell Physiol Biochem 2018;46:1536-1554 \begin{tabular}{l|l} 
and Biochemistry Published online: April 24, 2018 & O 2018 The Author \\
www.karger.com/cp
\end{tabular}

\section{Introduction}

Oxidative stress can result in extensive damage to cellular components including proteins, RNA, and DNA [1-3]. Reactive oxygen species (ROS) produced during oxidative stress mediate a wide range of pathological processes including lipid peroxidation, reduction of NO bioactivity, and induction of inflammatory genes [4]. Accumulating evidence suggests that oxidative stress represents a common pathogenic mechanism for neurodegenerative diseases [5-7], atherosclerosis [8], and diabetes [9]. In the central nervous system, excessive ROS accumulation may cause microglial cell activation, and neuron and astrocyte damage [10]. Parkinson's disease (PD), a chronic degenerative disease of the central nervous system, is pathologically characterized by the selective and progressive degeneration of dopaminergic neurons, and oxidative stress-induced neuronal apoptosis is implicated in this disease [5-7, 11-13].

Akt, also known as protein kinase B (PKB), is a serine/threonine protein kinase that regulates cell proliferation, migration, and apoptosis [14]. Several previous studies revealed that Akt signalling mediates neuronal survival, and the activation of Akt provides a neuroprotective effect in PD [15-17]. For example, activation of Akt/Rheb can induce central nervous system axon regeneration [18], and both total Akt and phosphorylation of Akt-S473 are greatly reduced in the brain tissue of Parkinson's patients [19]. Mechanistic studies suggest that Akt suppresses cell death through phosphorylating multiple apoptosis-related proteins [20]. Moreover, the PI3K/Akt activation may protect neural stem/progenitor cells against $\mathrm{H}_{2} \mathrm{O}_{2}$-evoked neurotoxicity, and $\mathrm{PI} 3 \mathrm{~K}$ inhibitors exaggerate $\mathrm{H}_{2} \mathrm{O}_{2}$-evoked SH-SY5Y cell death $[21,22]$.

Transcription factor EB (TFEB), a member of the MiT/TFE subfamily of basic helix-loophelix-leucine-zipper (bHLH-Zip) transcription factors, can enhance lysosomal biogenesis and autophagy [23-25] and ameliorate pathology of several human diseases, including neurodegenerative diseases [26], cancers [27-29], and lysosomal storage disorders [30]. Recent studies have demonstrated that activation of TFEB protects against cadmiuminduced neurotoxicity through enhancing lysosomal biogenesis and autophagic flux [31], and the cytoprotective effect of moderate levels of $\mathrm{Cu}$ exposure is related to the activation of TFEB [32]. Additionally, it is reported that TFEB overexpression can alleviate progression of Alzheimer's disease by reducing A $\beta$-induced ROS production and apoptosis [33] and that PGC-1alpha may rescue Huntington's disease proteotoxicity by preventing oxidative stress and promoting TFEB function [34]. Moreover, TFEB overexpression protects dopaminergic neurons under MPTP (1-methyl-4-phenyl-1, 2,3, 6-tetrahydropyridine)-induced oxidative stress [35]. Pharmacological activation of TFEB can improve lysosomal function, ameliorate $\alpha$-synuclein pathology in PD, and protect dopamine neurons against damage induced by oxidative stress and inflammation in PD [35-37]. Remarkably, Palmieri et al. recently reported that Akt phosphorylates TFEB at Ser467, and Akt-induced phosphorylation of TFEB prevents nuclear localization of TFEB, considerably inhibiting the transcription of TFEB-induced lysosomal genes, thus exerting a neuroprotective effect [38]. Although accumulating evidence supports that TFEB activation promotes cell survival under oxidative stress by regulating lysosome activity, whether the activation of TFEB exerts cytoprotective effects in a lysosome-independent pathway remain unanswered. In the present study, we show that a low concentration of $\mathrm{H}_{2} \mathrm{O}_{2}$ induced TFEB phosphorylation at Ser467 and nuclear translocation by activating Akt, facilitating neuronal survival, whereas a high concentration of $\mathrm{H}_{2} \mathrm{O}_{2}$ promoted SH-SY5Y cell apoptosis via suppressing TFEB-Ser467 phosphorylation and nuclear translocation.

\section{Materials and Methods}

Ethical Consent

All experiments were performed in accordance with the National Insititute of Health Guide for the Care and Use of Laboratory Animals, and were approved by the Animal Ethics Committee of Hebei Medical University, Shijiazhuang, China. 


\section{Cellular Physiology Cell Physiol Biochem 2018;46:1536-1554 \begin{tabular}{c|l} 
DOI: 10.1159/000489198 & $\begin{array}{l}\text { @ } 2018 \text { The Author(s). Published by S. Karger AG, Basel } \\
\text { www.karger.com/cpb }\end{array}$
\end{tabular}}

Su et al.: TFEB is Involved in $\mathrm{H}_{2} \mathrm{O}_{2}$-Induced Neuronal Apoptosis

Antibodies and reagents

Anti-PARP (46D11), anti-p-Akt-S473 (4058), anti-Akt (9272), anti-p-(Ser/Thr) Akt substrate (9611), anti-p44/42 MAPK (Erk1/2) (4696), anti-p-p44/42 MAPK (P-Erk1/2) (4377) and anti-HA (2999) antibodies were purchased from Cell Signaling Technology (Beverly, MA). Anti-GST (E022040) antibody was from EARTHOX (Millbrae, CA). The phospho-specific antibody of TFEB-S467 was generated and purified by Shanghai Abiocode Biotechnology (Shanghai, China). Anti-Flag (F1804), anti- $\beta$-actin (5316), dimethyl sulfoxide (DMSO) (D1435), Akt 1/2 kinase inhibitor (A6730), recombinant human EGF (E9644), hydrogen peroxide and 1-methyl-4-phenyl-1, 2,3, 6-tetrahydropyridine hydrochloride (MPTP) (M0896) were from Sigma (St. Louis, MO). Insulin was purchased from NovoNordisk. Wortmannin (681675) were from Merck Millipore (Kenilworth, NJ). PD98059 (1666) was from BioVision. Anti-LC3 (NB100-2220) was from NOVUS, and anti-SQSTM1/P62 (ab56416) was from Abcam.

\section{DNA construction}

The plasmids HA-Akt-WT/CA/KD/*KD and GST-Akt-PH/Cat/Tail were obtained from Prof. Keqiang Ye (Emory University, Atlanta, USA). Flag-TFEB was from Prof. Andrea Ballabio (Telethon Institute of Genetics and Medicine, Naples, Italy). The mutants (Flag-TFEB-S467A/D) were obtained by PCR mutagenesis. Wild type, fragments (1-319, 320-476, 320-335, 351-476), and the mutants (S462A, S463/467A, S466A, S467/469A) of TFEB were cloned into GST vector. TFEB, TFEB-S467A and TFEB-S467D were sub-cloned into pCDH-Flag vector.

\section{Cell culture and treatment}

The SH-SY5Y cell line, HEK293T were cultured in high glucose (25 mM) DMEM supplemented with 10\% fetal bovine serum, $100 \mathrm{U} / \mathrm{mL}$ penicillin, and $100 \mathrm{U} / \mathrm{mL}$ streptomycin. Hela stable cell lines were cultured in high glucose (25 mM) DMEM supplemented with 10\% fetal bovine serum, $100 \mathrm{U} / \mathrm{mL}$ penicillin, $100 \mathrm{U} /$ $\mathrm{mL}$ streptomycin, and $1 \mu \mathrm{g} / \mu \mathrm{L}$ puromycin. Recombinant DNA plasmids were transfected to 293T cells or SH-SY5Y cells with polyethylenimine. Cells were harvested after transfected $24 \mathrm{~h}$ for following experiments such as co-immunoprecipitation or Western blotting. The SH-SY5Y cells were infected with Lentivirus containing Flag/TFEB/TFEB-S467A/TFEB-S467D which was packaged into 293T. Then, the SH-SY5Y cells were pretreated with or without Akt $1 / 2$ kinase inhibitor for $1 \mathrm{~h}$, Wortmannin for $1 \mathrm{~h}$, and insulin/EGF for $15 \mathrm{~min}$, after that, treated with $\mathrm{H}_{2} \mathrm{O}_{2}$ for $24 \mathrm{~h}$. Primary neurons were prepared as previously reported [35]. Primary neurons were infected with adenovirus containing GFP/TFEB/TFEB-S467A/TFEB-S467D which were generated and purified by Shanghai Hanbio Biotechnology (Shanghai, China). Then, primary neurons were pretreated with or without Akt $1 / 2$ kinase inhibitor for $1 \mathrm{~h}$, after that, treated with $\mathrm{H}_{2} \mathrm{O}_{2}$ for $24 \mathrm{~h}$.

\section{Immunoprecipitation and Western blot}

Cells were lysed in lysis buffer (50 mM Tris-HCl, pH 7.4, $150 \mathrm{mM} \mathrm{NaCl,} 5$ mM EDTA, 1 mM PMSF) with a protease inhibitor and phosphatase inhibitor cocktails for $15 \mathrm{~min}$ on ice, then centrifuged at 12, $000 \mathrm{rpm}$ at $4^{\circ} \mathrm{C}$ for $15 \mathrm{~min}$. The supernatant was collected and boiled with SDS-sample buffer at $95^{\circ} \mathrm{C}$ for $10 \mathrm{~min}$. If the following experiment is immunoprecipitation, the supernatant was mixed with glutathione agarose for incubating $5 \mathrm{~h}$ or overnight at $4^{\circ} \mathrm{C}$. The complexes were washed 6 times with lysis buffer, and then boiled in SDS-sample buffer at $95^{\circ} \mathrm{C}$. The samples were separated by SDS-polyacrylamide gels and transferred to a PVDF membrane. The membrane was blocked in 5\% skim milk in TBST and incubated with the primary antibody overnight at $4^{\circ} \mathrm{C}$. The membrane was washed 3 times with TBST and incubated with secondary antibody for $2 \mathrm{~h}$ at room temperature. After washed 3 times with TBST, the membrane was incubated with ECL for 2 min in dark at room temperature. Then the chemiluminescent signals were captured by autoradiography films.

\section{Flow cytometry}

The SH-SY5Y cells and primary neurons were harvested after treatment with different conditions, and washed 2 times with PBS. The precipitation was resuspended with $100 \mu \mathrm{l} 1 \times$ binding buffer, and incubated with $5 \mu \mathrm{L}$ Annexin $\mathrm{V}$ and $5 \mu \mathrm{L}$ 7-AAD for $15 \mathrm{~min}$ at room temperature. Then the cellular fluorescence was measured by FACScan. The protocol was performed according to the procedure of PE Annexin V Apoptosis Detection Kit (BD Pharmingen 559763). Apoptosis was analyzed by flow cytometry (Guava easyCyte) for at least 10000 events as described. 


\section{Cellular Physiology Cell Physiol Biochem 2018;46:1536-1554 \begin{tabular}{c|l} 
DOI: 10.1159/000489198 & $\begin{array}{l}\text { ( ) } 2018 \text { The Author(s). Published by S. Karger AG, Basel } \\
\text { www.karger.com/cpb }\end{array}$
\end{tabular}}

Su et al.: TFEB is Involved in $\mathrm{H}_{2} \mathrm{O}_{2}$-Induced Neuronal Apoptosis

MTT assay

SH-SHY5Y cells were seeded to 96 -well culture plates at the density of $5 \times 10^{3} \sim 10^{4}$ per well. After cells were treated with different concentration of $\mathrm{H}_{2} \mathrm{O}_{2}$ for $24 \mathrm{~h}$, or cells were transfected with DNA plasmid HAvector, HA-Akt-CA, and HA-Akt-KD for $24 \mathrm{~h}$, cells were treated with hydrogen peroxide at the time course. Cells were added to $20 \mu \mathrm{L}$ MTT solution $\left(5 \mathrm{mg} / \mathrm{mL}\right.$ ) at per time point, and incubated for $4 \mathrm{~h}$ at $37{ }^{\circ} \mathrm{C}$. Medium was removed and cells were added $200 \mu \mathrm{L}$ DMSO to terminate reaction. The absorbance was obtained at a wavelength $570 \mathrm{~nm}$ by using ELISA plate reader (VERSAMax; Molecular Devices).

\section{In vitro kinase assay}

The 293T cells were harvested after transfected with Flag-TFEB/TFEB-S467A and HA-Akt-CA $24 \mathrm{~h}$. Cells were lysed $15 \mathrm{~min}$ on ice, and then centrifuged at $4^{\circ} \mathrm{C}$. The supernatants were mixed with anti-Flag M2 Magnetic Beads (Sigma, A2220) and anti-HA Magnetic Beads (Thermo, Scientific, 88836), respectively, and incubated for $5 \mathrm{~h}$ at $4^{\circ} \mathrm{C}$, and then the beads were washed 3 times with lysis buffer. The anti-flag beads containing the protein of flag-TFEB/TFEB-S467A were dephosphorylated by CIP enzyme at $37^{\circ} \mathrm{C}$ for $1 \mathrm{~h}$, and washed 3 times with lysis buffer and 3 times with Akt kinase assay buffer ( $25 \mathrm{mM}$ Tris-HCl, PH7.5, 5 $\mathrm{mM} \beta$-glycerolphosphate, $2 \mathrm{mM}$ DTT, $0.1 \mathrm{mM} \mathrm{Na}_{3} \mathrm{VO}_{4}, 10 \mathrm{mM} \mathrm{MgCl}_{2}$, and $200 \mu \mathrm{M}$ ATP). The anti-Flag beads containing the dephosphorylated protein of Flag-TFEB/TFEB-S467A were resuspended with $40 \mu \mathrm{L}$ Akt kinase assay buffer, and mixed with anti-HA beads containing active Akt protein and incubated at $37^{\circ} \mathrm{C}$ for $1 \mathrm{~h}$. The complexes were washed with lysis buffer 3 times, and boiled with SDS loading buffer at $100^{\circ} \mathrm{C}$ for $10 \mathrm{~min}$. The phosphorylated protein of TFEB was detected by the specific antibody against p-TFEB-S467.

\section{Nuclear extraction}

SH-SHY5Y cells were treated with different concentration of $\mathrm{H}_{2} \mathrm{O}_{2}$ for $24 \mathrm{~h}$ or infected with Lentivirus containing Flag-TFEB-S467A/D for $48 \mathrm{~h}$ and treated with different concentration of $\mathrm{H}_{2} \mathrm{O}_{2}$ for $24 \mathrm{~h}$. The medium was removed, and cells were washed 3 times by cold PBS. Then added 500 ? $\mathrm{L}$ of nuclear extraction solution (10 mM HEPES (pH 7.9), $10 \mathrm{mM} \mathrm{KCl,} 2 \mathrm{mM} \mathrm{MgCl}_{2}, 0.5 \mathrm{mM}$ DTT, and 0.1\% NP-40) and cells were scraped off from the dishes and transferred to a microcentrifuge tube, and incubated for 20 min on ice. Cell suspensions were centrifuged at $3,000 \mathrm{rpm}$ at $4{ }^{\circ} \mathrm{C}$. The supernatant was saved as the cytosolic fraction. The precipitate was washed 3 times by PBS, then was resuspended with lysis buffer (20 mM HEPES (pH 7.9), $0.2 \mathrm{mM}$ EDTA, $1.5 \mathrm{mM} \mathrm{MgCl}_{2}, 0.42 \mathrm{mM} \mathrm{NaCl}, 0.5 \mathrm{mM}$ DTT, and 25\% glycerol with phosphatase inhibitors: $1 \mathrm{mM} \mathrm{Na}_{3} \mathrm{VO}_{4}, 1 \mathrm{mM} \mathrm{NaF}, 100 \mu \mathrm{M} \beta$-glycerophosphate) and incubated for $1 \mathrm{~h}$ on ice. The suspensions were centrifuged at $12,000 \times \mathrm{g}$ for $15 \mathrm{~min}$, and the supernatant was collected as the nuclear fraction.

\section{Immunofluorescence assay}

SHY5Y cells or stable cell line expressing Flag-TFEB-S467A/D were grown on glass coverslips for $24 \mathrm{~h}$ and treated with $\mathrm{H}_{2} \mathrm{O}_{2}$. The cells were washed with PBS, and fixed with $4 \%$ paraformaldehyde (PFA) for 15 min. Then washed 3 times with PBS, and treated with $0.5 \%$ Triton-X 100 for 15 min. The coverslips were washed with PBS once again, and were blocked with blocking buffer (3\% BSA in PBS) for $1 \mathrm{~h}$ and incubated with appropriate primary antibodies (1:100) at $4^{\circ} \mathrm{C}$ overnight. After 3 times washed with PBS, the coverslips were incubated with appropriate secondary antibodies (1:500) for $1 \mathrm{~h}$ at room temperature and finally incubated in DAPI for 2 min. Coverslips were mounted on a glass slide with Fluoromount (Sigma), and images were obtained using a confocal microscope (Olympus FV1200).

\section{Reverse transcriptase-PCR analysis}

Total RNA were extracted from CTR, TFEB, TFEB-S467A and TFEB-S467D overexpressing SH-SHY5Y cells treated with $\mathrm{H}_{2} \mathrm{O}_{2}$ using RNAiso Plus (TaKaRa) according to the manufacturer's instructions. One microgram of RNA was

Table 1. RT primers

\begin{tabular}{lr}
\hline Gene & Sequence \\
\hline GALNS-F & 5'-TTGTCGGCAAGTGGCATCT-3' \\
GALNS-R & 5'-CCAAACCACTCATCAAATCCG-3' \\
GBA-F & 5'-TGGGTACCCGGATGATGTTA-3' \\
GBA-R & 5'-AGATGCTGCTGCTCTCAACA-3' \\
GLA-F & 5'-AGCCAGATTCCTGCATCAGTG-3' \\
GLA-R & 5'-ATAACCTGCATCCTTCCAGCC-3' \\
CLCN7-F & 5'-TGATCTCCACGTTCACCCTGA-3' \\
CLCN7-R & 5'-TCTCCGAGTCAAACCTTCCGA-3' \\
CTSB-F & 5'-AGTGGAGAATGGCACACCCTA-3' \\
CTSB-R & 5'-AAGAAGCCATTGTCACCCCA-3' \\
ARSA-F & 5'-AGAGCTTTGCAGAGCGTTCAG-3' \\
ARSA-R & 5'-ATACGCATGGTCTCAGGTCCA-3' \\
ATP6V1H-F & 5'-GGAAGTGTCAGATGATCCCCA-3' \\
ATP6V1H-R & 5'-CCGTTTGCCTCGTGGATAAT-3' \\
\hline
\end{tabular}




\section{Cellular Physiology Cell Physiol Biochem 2018;46:1536-1554 \begin{tabular}{l|l} 
and Biochemistry $10.1159 / 000489198$ & $\begin{array}{l}\text { O 2018 The Author(s). Published by S. Karger AG, Basel } \\
\text { www.karger.com/cpb }\end{array}$ \\
\hline
\end{tabular} \\ Su et al.: TFEB is Involved in $\mathrm{H}_{2} \mathrm{O}_{2}$-Induced Neuronal Apoptosis}

used as a template for quantitative reverse transcriptase- (RT-) PCR amplification using One Step SYBR ${ }^{\circledR}$ PrimeScrip $^{\text {TM }}$ RT-PCR Kit (TaKaRa). Quantitative real-time PCR were performed by using an ABI 7900HT Real-Time PCR System with SYBR ${ }^{\circledast}$ Premix Ex Taq ${ }^{\mathrm{TM}}$ II (TaKaRa). The relative abundance of transcripts was calculated based on normalization to the GAPDH gene. The primers are shown in Table 1.

\section{Animals and treatment}

Eight-week-old male C57BL/6 mice weighing approximately 25 g purchased from SLRC Laboratory. These mice were treated by the following procedures: the left substantia nigra pars compacta (anteriorposterior $-3.1 \mathrm{~mm}$, medial-lateral $-1.2 \mathrm{~mm}$, and dorsal-ventral $-4.6 \mathrm{~mm}$, as measured from the bregma) [35] of mice were injected $1 \mu \mathrm{L}$ adenovirus ( $\mathrm{n}=6$ mice per group) by the surgery of brain stereotaxis using the apparatus purchased from Narishige Scientific Instrument, then the mice were injected with MPTP (20 $\mathrm{mg} / \mathrm{kg} /$ day) intraperitoneally (i.p) for 10 days after stereotaxic injection 3 days.

\section{TH staining}

The mice were anesthetized and then perfused 4\% paraformaldehyde after PBS perfusion. The whole brain of mouse was removed into $4 \%$ paraformaldehyde for $5 \mathrm{~h}$, and dehydrated in $30 \%$ sucrose overnight. The brain slice which is $10 \mu \mathrm{m}$ thickness was washed with PBS, fixed in 4\% paraformaldehyde 15 min after washed 3 times with PBS, and permeabilized with $0.5 \%$ Triton-X 100 in PBS for 15 min. The slice was blocked with $3 \%$ normal goat serum in PBS for $1 \mathrm{~h}$, and then washed 3 times with PBS. The slice was incubated with tyrosine hydroxylase antibody (1:200) at room temperature overnight, and washed 3 times with PBS. Then, the slice was incubated with the secondary antibody (Invitrogen, A11004). Last, the slice was dyed with DAPI for 2 min. The counts of TH positive cells were quantified by unbiased sterological analytic system NewCAST.

\section{Statistical analysis}

Statistical analysis of the data were performed with Graphpad Prism 5.0 software. Data are expressed as mean \pm standard error of mean (SEM) from three independent experiments. Data were analyzed by one-way ANOVA. Newman-Keuls post hoc analysis was employed when differences in ANOVA testing were observed $(\mathrm{P}<0.05)$.

\section{Results}

Suppression of Akt signalling is responsible for H2O2-induced apoptosis in SH-SY5Y cells

Because Akt is known to mediate growth factor-induced neuronal survival [15], and because excessive ROS can cause damage of neuronal cells [39], we first tested whether inhibition of Akt signalling is responsible for $\mathrm{H}_{2} \mathrm{O}_{2}$-induced SH-SY5Y cell apoptosis. SHSY5Y cells were treated with different concentrations of $\mathrm{H}_{2} \mathrm{O}_{2}$. The MTT assay showed that although $\mathrm{H}_{2} \mathrm{O}_{2}$ at concentrations of $100 \mu \mathrm{M}$ and $200 \mu \mathrm{M}$ did not significantly affect SH-SY5Y cell viability, exposure of cells to 400 or $800 \mu \mathrm{M} \mathrm{H}_{2} \mathrm{O}_{2}$ markedly decreased cell viability (Fig. 1A). Western blot analysis showed that a low concentration of $\mathrm{H}_{2} \mathrm{O}_{2}(100 \mu \mathrm{M}$ and 200 $\mu \mathrm{M})$ moderately increased Akt phosphorylation, whereas the level of phosphorylated Akt decreased when the SH-SY5Y cells were treated with a high concentration of $\mathrm{H}_{2} \mathrm{O}_{2}(400 \mu \mathrm{M}$ and $800 \mu \mathrm{M}$ ) (Fig. 1B), indicating that different concentrations of $\mathrm{H}_{2} \mathrm{O}_{2}$ have different effects on Akt phosphorylation and cell viability.

A previous study reported that levels of only total Akt and active phospho-Ser473-Akt was diminished in the brain in PD patients [19]. As shown in Fig. 1B, the trend of pAkt Thr308 expression was same as that of pAkt Ser473 expression when SH-SHY5Y cells were exposed to different concentrations of $\mathrm{H}_{2} \mathrm{O}_{2}$. Therefore, in the following experiments, the phosphoSer473-Akt expression was detected only under oxidative stress. Next, the SH-SY5Y cells were pretreated with an Akt inhibitor or Wortmannin, a PI3K inhibitor, and then exposed to $400 \mu \mathrm{M} \mathrm{H}_{2} \mathrm{O}_{2}$ for $24 \mathrm{~h}$. Flow cytometric analysis showed that inhibition of Akt signalling by the Akt or PI3K inhibitor further increased the cell apoptosis induced by oxidative stress (Fig. 1C). A similar result was obtained by Western blotting, showing that compared with 
Fig. 1. Suppression of the Akt signaling is responsible for $\mathrm{H}_{2} \mathrm{O}_{2}$-induced apoptosis in SH-SY5Y cells. (A, B) SH-SY5Y cells were treated with the indicated concentrations of $\mathrm{H}_{2} \mathrm{O}_{2}$ for $24 \mathrm{~h}$. Cell viability was measured by MTT assay, values are the mean \pm SEM. $* P<0.05$, $* * P<0.01$ compared with the control group (A). Akt phosphorylation was analyzed by Western blotting with anti-p-Akt-S473/Thr308 antibody, whereas band intensities that were normalized to $\beta$-actin are shown on the right, values are the mean \pm SEM of 3 independent experiments. $* P<0.05, \quad * * P<0.01$ versus control group (B). (C, D) SH-SY5Y cells were pretreated with DMSO, Akt inhibitor (Akt1/2 kinase inhibitor, $4 \mu \mathrm{M}$ ), or Wortmannin $(0.1 \mu \mathrm{M})$ for $1 \mathrm{~h}$, and then cells were treated with $\mathrm{H}_{2} \mathrm{O}_{2}(400$ $\mu \mathrm{M}$ ) for $24 \mathrm{~h}$. Cell apoptosis was measured by flow cytometry. The $\mathrm{X}$-axis represents the fluorescence intensity of Annexin-V-PE, and the $\mathrm{y}$-axis represents the fluorescence intensity of 7-AAD. The data

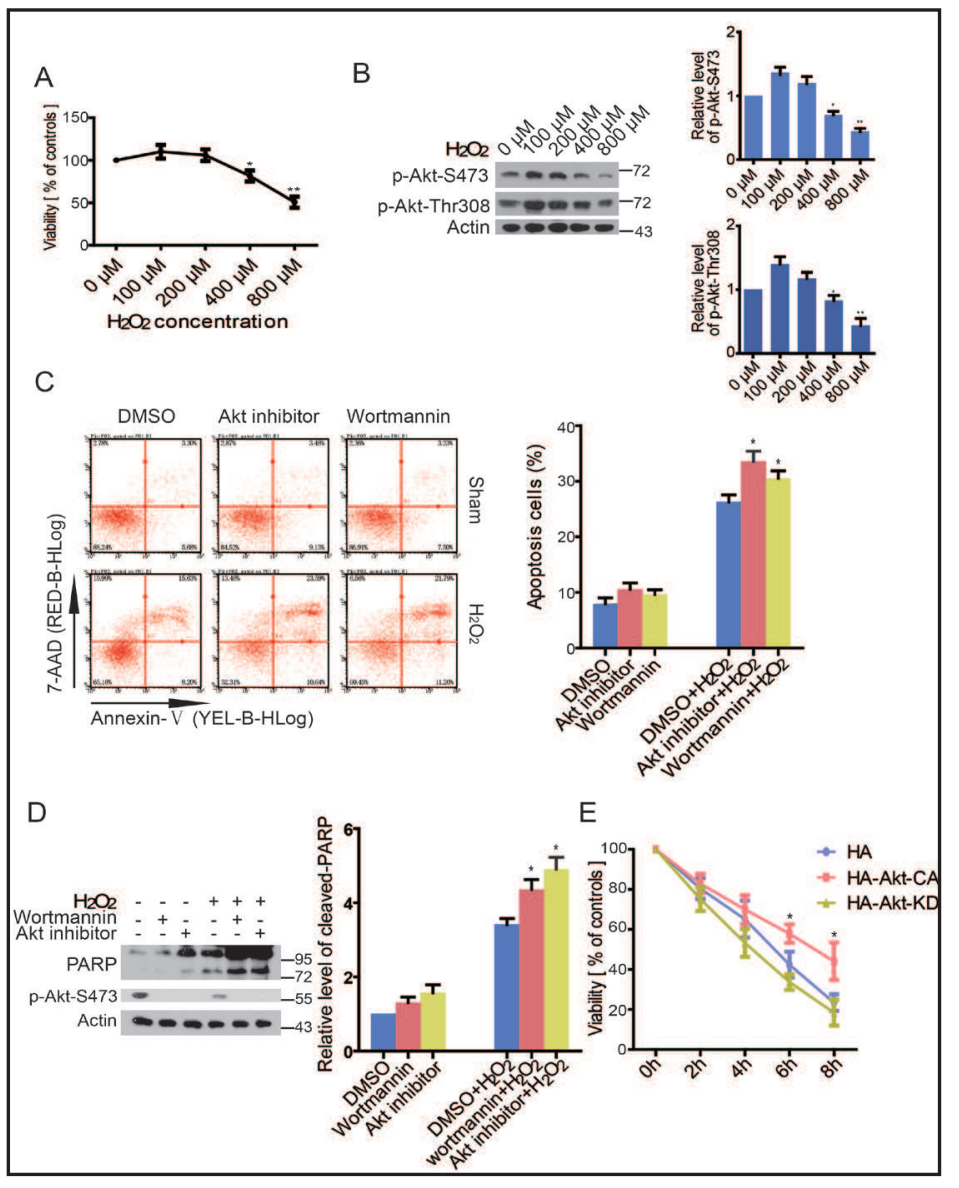
of flow cytometry were quantified are shown on the right, values are the mean \pm SEM. $* P<0.05$ versus DMSO $+\mathrm{H}_{2} \mathrm{O}_{2}$ group (C). PARP cleavage and Akt phosphorylation were analyzed by Western blotting, whereas band intensities that were normalized to $\beta$-actin are shown on the right, values are the mean \pm SEM of 3 independent experiments. $* P<0.05$ versus DMSO $+\mathrm{H}_{2} \mathrm{O}_{2}$ group (D). (E) The restructured plasmids HA-vector, HA-Akt-CA (constitutively active), or HA-Akt-KD (kinase-dead) were transfected into SH-SY5Y cells. Cells were treated with $\mathrm{H}_{2} \mathrm{O}_{2}(400 \mu \mathrm{M})$ for the indicated times, and the cell viability was estimated by MTT assay. Values are the mean \pm SEM. $* P<0.05$ versus HA group.

$\mathrm{H}_{2} \mathrm{O}_{2}$ alone, Akt or PI3K inhibitor completely blocked Akt phosphorylation and further increased $\mathrm{H}_{2} \mathrm{O}_{2}$-induced PARP cleavage, a hallmark of apoptosis (Fig. 1D). Further, SH-SY5Y cells were transfected with a plasmid vector encoding constitutively active Akt (HA-Akt-CA) or its kinase-dead mutant (HA-Akt-KD) and were treated with $\mathrm{H}_{2} \mathrm{O}_{2}$. MTT assay indicated that $\mathrm{H}_{2} \mathrm{O}_{2}$-reduced cell viability was rescued in HA-Akt-CA-transfected SH-SY5Y cells (Fig. 1E). Taken together, these findings clearly suggest that inhibition of Akt signalling is responsible for neuronal cell apoptosis induced by oxidative stress.

\section{TFEB-suppressed apoptosis of SH-SY5Y cells is affected by Akt signalling}

Because TFEB is known to suppress cell apoptosis [35], we sought to determine whether $\mathrm{H}_{2} \mathrm{O}_{2}$-induced SH-SY5Y cell apoptosis is affected by TFEB. Thus, SH-SY5Y cells were infected with lentivirus encoding Flag-TFEB, and cell apoptosis was detected by Western blot analysis and flow cytometry. $\mathrm{H}_{2} \mathrm{O}_{2}$-induced cleavage of PARP was markedly decreased in the TFEBoverexpressing cells (Fig. 2A, lane 4 versus lane 3), and blockage of Akt signalling by an Akt inhibitor enhanced the cleavage of PARP suppressed by TFEB overexpression (Fig. 2B, lane 4 versus lane 2). Similarly, Akt signalling activation by epidermal growth factor (EGF) further decreased $\mathrm{H}_{2} \mathrm{O}_{2}$-induced cleavage of PARP in the TFEB-overexpressing cells (Fig. 2C, lane 4 


\section{Cellular Physiology \\ Cell Physiol Biochem 2018;46:1536-1554 and Biochemistry

Fig. 2. TFEB-suppressed apoptosis of SH-SY5Y cells is affected by Akt signaling. (A) SH-SY5Y cells were infected with lentiviruses Flagvector (CTR) or Flag-TFEB for 48 $h$, and then treated with $\mathrm{H}_{2} \mathrm{O}_{2}(400$ $\mu \mathrm{M}$ ) for $24 \mathrm{~h}$. PARP cleavage and Akt phosphorylation were analyzed by Western blotting, whereas band intensities normalized to $\beta$-actin are shown on the right, values are the mean \pm SEM of 3 independent experiments. $* P<0.05$ versus corresponding CTR group, ${ }^{\#} P<0.05$ versus TFEB group. $(\mathrm{B}, \mathrm{C})$ SH-SY5Y cells were infected with lentiviruses Flag-vector (CTR) or Flag-TFEB for $48 \mathrm{~h}$, and pretreated with Akt inhibitor (Akt1/2 kinase inhibitor, $4 \mu \mathrm{M}$ ) for $1 \mathrm{~h}$ or with EGF (100 ng/mL) for $15 \mathrm{~min}$, and then treated with $\mathrm{H}_{2} \mathrm{O}_{2}(400 \mu \mathrm{M})$ for $24 \mathrm{~h}$. PARP cleavage and Akt phosphorylation were analyzed by Western blotting, whereas band intensities normalized to $\beta$-actin are shown on the right, values are the mean \pm SEM of 3 independent experiments. $* P<0.05$ versus corresponding CTR group, ${ }^{*} P<0.05$ versus TFEB $+\mathrm{H}_{2} \mathrm{O}_{2}$ group. (D) $\mathrm{SH}-$

SY5Y cells were infected with lentiviruses Flag-vector (CTR) or Flag-TFEB for $48 \mathrm{~h}$, and pretreated with Akt inhibitor (Akt1/2 kinase inhibitor, $4 \mu \mathrm{M})$ for $1 \mathrm{~h}$ or with EGF $(100 \mathrm{ng} / \mathrm{mL})$ for $15 \mathrm{~min}$, and then treated with $\mathrm{H}_{2} \mathrm{O}_{2}(400 \mu \mathrm{M})$ for $24 \mathrm{~h}$. Cell apoptosis was detected by flow cytometry. The x-axis represents the fluorescence intensity of Annexin-V-PE, and the y-axis represents the fluorescence intensity of 7-AAD. The data of flow cytometry were quantified are shown on the right. Values are the mean \pm SEM. $* P<0.05$ versus their corresponding CTR group, ${ }^{\#} P<0.05$ versus TFEB $+\mathrm{H}_{2} \mathrm{O}_{2}$ group.

versus lane 3). TFEB overexpression significantly decreased cell apoptosis regardless of the presence or absence of $\mathrm{H}_{2} \mathrm{O}_{2}$ (Fig. 2D).

To further test whether TFEB-suppressed cell apoptosis is affected by Akt signalling, TFEB-overexpressing SH-SY5Y cells were pretreated with an Akt inhibitor or EGF, an Akt activator [40], prior to $\mathrm{H}_{2} \mathrm{O}_{2}$ treatment. The results showed that TFEB overexpression significantly reduced the apoptosis induced by the Akt inhibitor and $\mathrm{H}_{2} \mathrm{O}_{2}$. Conversely, Akt signalling activation by EGF facilitated cell survival under oxidative stress, and TFEB overexpression further decreased $\mathrm{H}_{2} \mathrm{O}_{2}$-induced apoptosis in EGF-treated cells (Fig. 2D). These results indicated that TFEB-suppressed SH-SY5Y cell apoptosis is affected by Akt signalling.

\section{TFEB interacts with Akt}

Because we have found that TFEB-suppressed apoptosis of SH-SY5Y cells was affected by Akt signalling, we sought to reveal whether Akt directly interacted with TFEB. Thus, GST pull down with overexpressed wild-type Akt (HA-Akt-WT) and GST-TFEB was performed in 


\section{Cellular Physiology Cell Physiol Biochem 2018;46:1536-1554 \begin{tabular}{c|l} 
DOI: 10.1159/000489198 & $\begin{array}{l}\text { ( ) } 2018 \text { The Author(s). Published by S. Karger AG, Basel } \\
\text { www.karger.com/cpb }\end{array}$
\end{tabular} \\ Su et al.: TFEB is Involved in $\mathrm{H}_{2} \mathrm{O}_{2}$-Induced Neuronal Apoptosis}

Fig. 3. TFEB interacts with Akt. (A) HEK293T cells were co-transfected with MGST, or MGST-TFEB and HA-Akt-WT for $24 \mathrm{~h}$. MGST-TFEB was pulled down by using glutathione agarose beads, and proteins on the beads were detected by Western blotting with the indicated antibodies. (B) HEK293T cell lysates were incubated with IgG-Protein A/G beads or TFEB-Protein A/G beads, respectively. TFEB was pulled down with anti-TFEB antibody, and Akt was detected by Western blotting. (C) HEK293T cells were cotransfected with MGST-TFEB-FL, MGSTTFEB-1-294, or MGST-TFEB-295-476 and HA-Akt-WT for $24 \mathrm{~h}$, and the different regions of TFEB interacted with Akt were detected by Western blotting with the indicated antibodies. (D) HEK293T cells were co-transfected with MGSTTFEB and HA-Akt-WT/*KD (partial kinase dead)/CA (constitutively active)/ KD (kinase dead) for $24 \mathrm{~h}$, and the different activity forms of Akt interacted with TFEB were detected by Western blotting with the indicated antibodies. (E)
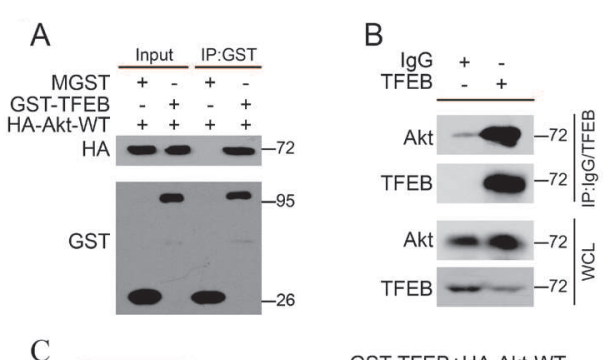

C

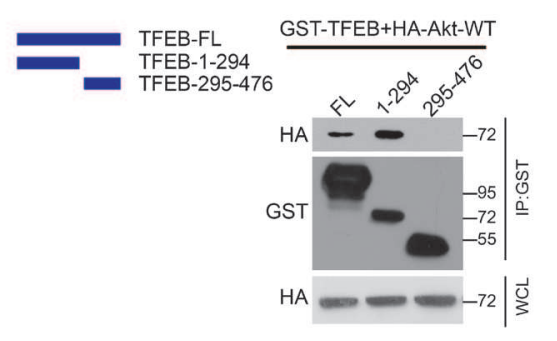

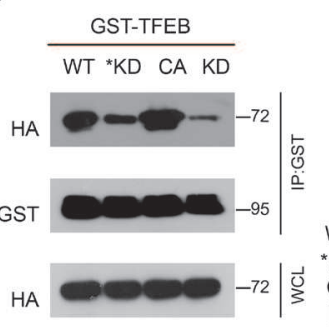

$E$
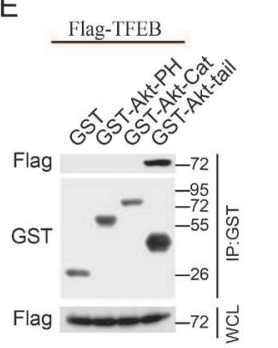
HEK293T cells were co-transfected with

Flag-TFEB and MGST or GST-Akt-PH/Cat/Tail for $24 \mathrm{~h}$, and the different domains of Akt interacted with TFEB were detected by Western blotting with the indicated antibodies.

vitro. Apparently, HA-Akt physically bound to GST-TFEB in vitro but not GST alone (Fig. 3A). The interaction of Akt with TFEB was additionally identified by a co-immunoprecipitation assay with endogenous proteins, which demonstrated that Akt is present in the anti-TFEB immunoprecipitates (Fig. 3B). To further define the domain of TFEB required for binding to Akt, HEK293T cells were co-transfected with GST-TFEB-FL, GST-TFEB-1-294 or GSTTFEB-295-476 and HA-Akt-WT. Full-length TFEB (TFEB-FL) and various truncated mutants were tested for their ability to interact with Akt using GST pull-down assays. The results showed that the full-length TFEB and its N-terminal region (TFEB-1-294), which contains a HLH domain, interacted with Akt (Fig. 3C). When the N-terminal part (amino acids 1-294) of TFEB was deleted, the interaction of the mutant with Akt was abolished (Fig. 3C).

To determine whether TFEB and Akt interaction was influenced by Akt activity, HEK293T cells were co-transfected with a plasmid vector encoding wild-type Akt (HA-Akt-WT), constitutively active Akt (HA-Akt-CA), kinase-dead Akt (HA-Akt-KD) or partial kinase-dead Akt (HA-Akt-*KD) and GST-TFEB, and interaction of TFEB with the different activity forms of Akt was examined. GST pull-down assays showed a stronger binding of constitutively active Akt to TFEB, and kinase-dead Akt almost completely lost its TFEB-binding activity (Fig. 3D). Further, the different domains of Akt were evaluated for their ability to bind TFEB. The results showed that TFEB interacted with Akt-tail but not Akt-PH or Akt-Cat (Fig. 3E). All these data suggested that TFEB and Akt associate with each other and that their association is mediated through the HLH domain of TFEB and Akt-tail.

TFEB is phosphorylated by Akt

The results that TFEB and Akt associate with each other prompted us to investigate whether Akt can phosphorylate TFEB. Thus, stable cell lines expressing Flag-TFEB were 


\section{Cellular Physiology \\ Cell Physiol Biochem 2018;46:1536-1554 and Biochemistry

Fig. 4. TFEB is phosphorylated by Akt signaling. (A) HEK293T cells stablely expressing Flag-TFEB were treated with either the PI3K inhibitor wortmannin, Akt inhibitor, or ERK inhibitor PD98059 in the presence or absence of EGF. The phosphorylation of Akt substrate, Akt and ERK was detected by Western blotting. For detecting p-Akt substrate, the cell lysates were immunoprecipitated with anti-Flag antibody prior to testing. (B) HEK293T cells were cotransfected with MGST-TFEB and HA-vector or HA-Akt-WT/CA/KD for $24 \mathrm{~h}$, and Akt substrate phosphorylation and Akt interaction with its substrate were detected by Western blotting. For detecting pAkt substrate, the cell lysates were pulled down by using glutathione agarose beads prior to testing. (C) HEK293T cells were transfected with MGST-TFEB for $24 \mathrm{~h}$, and then treated with or without EGF. The cell lysates were pulled down
A

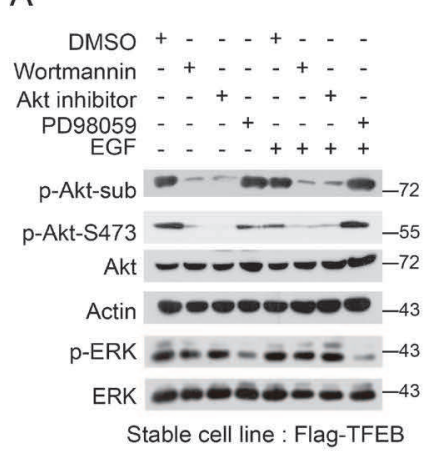

C

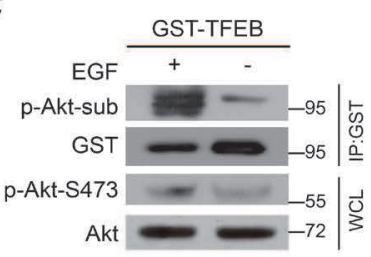

$E$

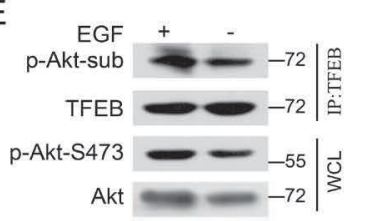

B

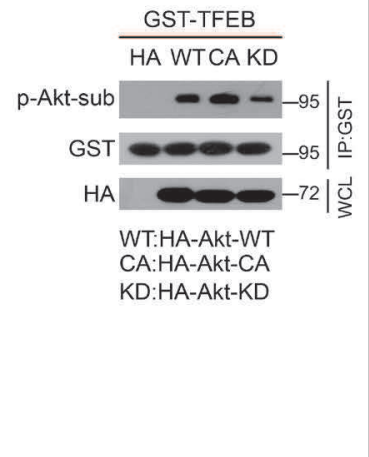

D

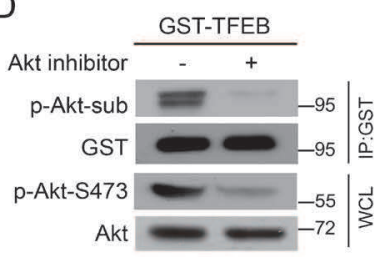

$\mathrm{F}$

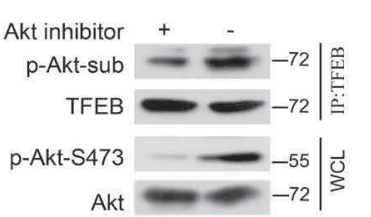

by using glutathione agarose beads, and proteins on the beads were detected by Western blotting. (D) HEK293T cells were transfected with MGST-TFEB for $24 \mathrm{~h}$, and then treated with or without Akt inhibitor. The following experiments were performed as described in (C). (E, F) HEK293T cells were treated with or without EGF/Akt inhibitor, and the cell lysates were immunoprecipitated with anti-TFEB antibody, and the phosphorylation of Akt substrate and Akt-S473 was detected by Western blotting.

incubated with either the PI3K kinase inhibitor Wortmannin, the Akt inhibitor, or the ERK inhibitor PD98059 and then treated with EGF to activate Akt. TFEB phosphorylation was determined with an anti-p-Akt-substrate antibody. Western blot analysis showed that the phosphorylation of Akt-Ser473 and Akt-substrate markedly increased in response to insulin. Pharmacological inhibition of Akt or PI3K blocked the phosphorylation of Akt-Ser473 and Akt-substrate regardless of the presence or absence of insulin, while the ERK inhibitor did not affect their phosphorylation (Fig. 4A). We next examined whether the different activity forms of Akt also affected Akt-substrate phosphorylation, and found that constitutively active Akt had a stronger binding capability for Akt-substrate than wild-type Akt and kinase-dead Akt, as shown by GST pull-down assays (Fig. 4B). In further experiments, HEK293T cells were transfected with GST-TFEB and then treated with EGF or an Akt inhibitor, respectively. The results showed that EGF treatment promoted and the Akt inhibitor suppressed the phosphorylation of Akt-Ser473 and Akt-substrate (Fig. 4C and D). A similar result was obtained by pulling down endogenous TFEB from HEK293T cells treated with an Akt activator or inhibitor (Fig. 4E and F). Together, the above results clearly suggested that TFEB is phosphorylated by Akt.

\section{Akt phosphorylates TFEB at Ser467}

Because there are several putative Akt phosphorylation sites in TFEB, we sought to verify which sites are phosphorylated by Akt. First, the different TFEB-truncated mutants were generated (Fig. 5A), and their phosphorylation by Akt was determined using anti-p- 
Fig. 5. Akt phosphorylates TFEB at Ser467. (A) Schematic diagram of full-length TFEB and its various truncated mutants. (B) HEK293T cells were co-transfected with MGST-TFEB-FL, MGST-TFEB-1-319 or MGST-TFEB-320-476 and HA-Akt-CA for $24 \mathrm{~h}$. Cell lysates were pulled down by using glutathione agarose beads, and proteins on the beads were detected by Western blotting with the indicated antibodies. (C) HEK293T cells were co-transfected with MGST-TFEB-320-476, MGST-TFEB-320-461 or MGSTTFEB-352-476 and HA-Akt-CA for $24 \mathrm{~h}$. The following experiments were performed as described in (B). (D) HEK293T cells were cotransfected with the different TFEB phosphorylation-deficient mutants and HA-Akt-CA for $24 \mathrm{~h}$. The following experiments were performed as described in (B). (E) HEK293T cells were co-transfected with Flag-TFEB or Flag-TFEBS467A and HA-Akt-CA for $24 \mathrm{~h}$. Cell

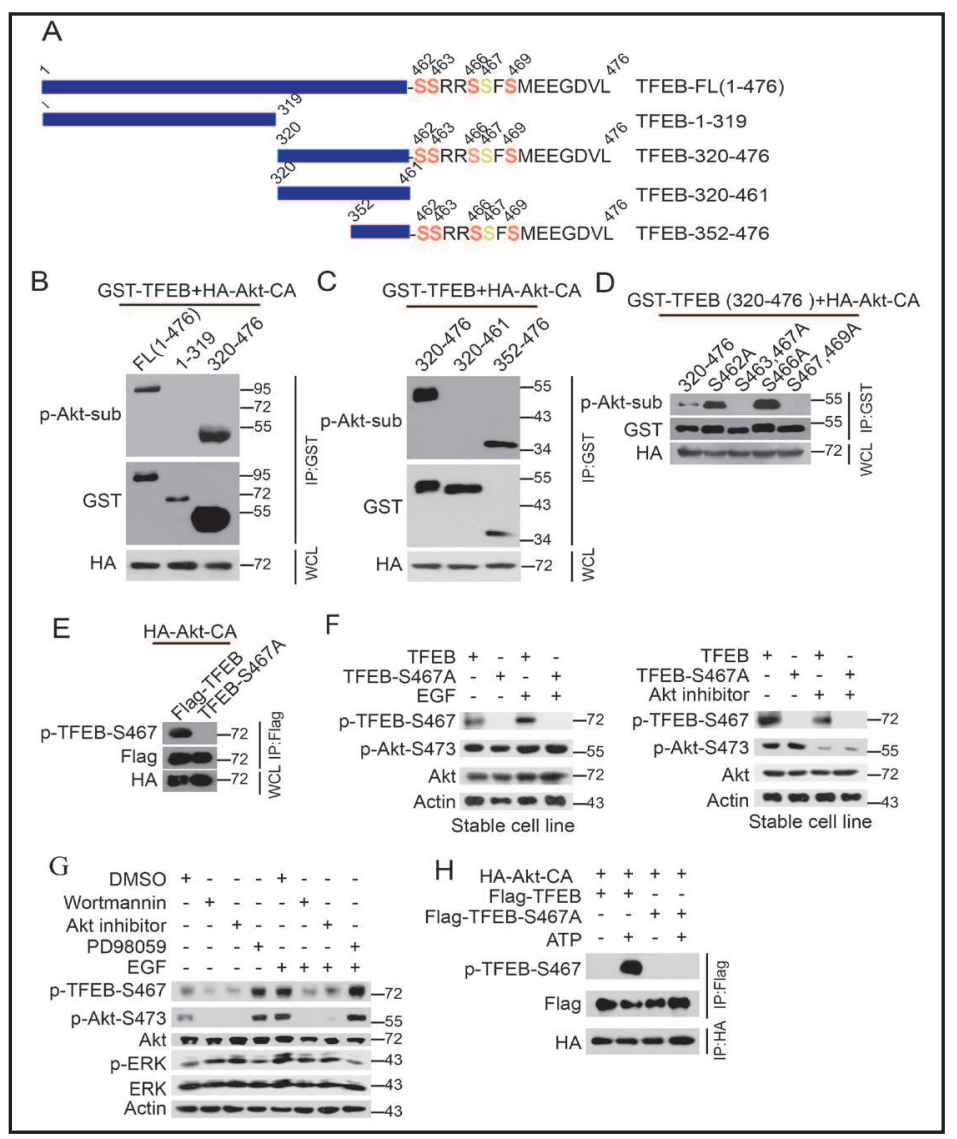
lysates were incubated with Flag beads, and the phosphorylation of TFEB-S467 was detected by Western blotting with phospho-specific antibody against TFEB-S467. (F) The stable cell line expressing Flag-TFEB or Flag-TFEB-S467A was treated with or without EGF/Akt inhibitor. The phosphorylation of TFEB-S467 and Akt-S473 was detected by Western blotting with the indicated antibodies. (G) SH-SY5Y cells were treated with either the Wortmannin, Akt inhibitor, or PD98059 in the presence or absence of EGF. The phosphorylation of TFEB-S467, Akt, and ERK was detected by Western blotting with the indicated antibodies. (H) In vitro Akt kinase assay. The TFEB-WT and TFEB-S467A purified by Flag pull down were incubated with the Akt-CA for $30 \mathrm{~min}$ in vitro, and the phosphorylation of TFEB-S467 was detected by Western blotting with phospho-specific antibody against TFEB-S467.

Akt-sub antibody. As shown in Fig. 5B, full-length TFEB (TFEB-FL) and its C-terminal part (TFEB-320-476, amino acids 320-476) were phosphorylated by Akt; however, the N-terminal part (TFEB-1-319, amino acids 1-319) was not phosphorylated, suggesting that TFEB-320476 contains Akt phosphorylation sites. When TFEB-320-476 was further truncated, we found that TFEB-352-476 (amino acids 352-476) could be phosphorylated by Akt, whereas TFEB-320-461 (amino acids 320-461) could not be phosphorylated (Fig. 5C), indicating that the serines between the $461^{\text {th }}$ and $476^{\text {th }}$ amino acids in TFEB are necessary for the phosphorylation of TFEB by Akt. Next, we generated point mutations of the serines between the $461^{\text {th }}$ and $476^{\text {th }}$ amino acids. Immunoblot analysis with an anti-p-Akt-sub antibody showed that the phosphorylation-deficient TFEB mutants, TFEB-S462A and TFEB-S466A, were phosphorylated by Akt at levels comparable to those of wild-type TFEB. By contrast, TFEB-S463/467A and TFEB-S467/469A mutants could not be phosphorylated by Akt (Fig. 5D). These findings suggested that Ser467 is necessary for the phosphorylation of TFEB by Akt.

To further substantiate the importance of Ser467 in the phosphorylation of TFEB by Akt, we generated a phospho-specific antibody against TFEB-S467 and further examined 


\section{Cellular Physiology \begin{tabular}{ll|l} 
and Biochemistry & Dublished online: April 24, 2018 & $\begin{array}{l}\text { O 2018 The Author(s). Published by S. Karger AG, Basel } \\
\text { www.karger.com/cpb }\end{array}$ \\
\cline { 1 - 2 }
\end{tabular}}

Su et al.: TFEB is Involved in $\mathrm{H}_{2} \mathrm{O}_{2}$-Induced Neuronal Apoptosis

Fig. 6. Phosphorylated TFEB translocates into the nucleus but not regulates lysosome gene expression under oxidative stress. (A, B) SH-SY5Y cells were treated with the indicated concentrations of $\mathrm{H}_{2} \mathrm{O}_{2}$ for $24 \mathrm{~h}$. The subcellular fractions were separated, p-TFEBS467 in the cytoplasm and nucleus was analyzed by Western blotting, whereas band intensities normalized to lamin B or $\alpha$-tublin are shown on the right, values are the mean \pm SEM of 3 independent experiments. $* P<0.05$ versus $0 \mu \mathrm{M}$ $\mathrm{H}_{2} \mathrm{O}_{2}$ group (A). The localization of p-TFEB-S467 was detected by immunofluorescence staining and observed via confocal microscopy. The percentage of TFEB-positive nuclei in SH-SY5Y cells is shown below the immunofluorescence staining. The data represent mean \pm SEM of 3 independent experiments in which 100 cells were analyzed. $* P<0.05$ versus $0 \mu \mathrm{M}$ $\mathrm{H}_{2} \mathrm{O}_{2}$ group (B). (C, D, E) SH-SY5Y cells were infected with lentiviruses Flag-vector (CTR), Flag-TFEB, Flag-TFEB-S467A or Flag-TFEBS467D for $48 \mathrm{~h}$, and then treated

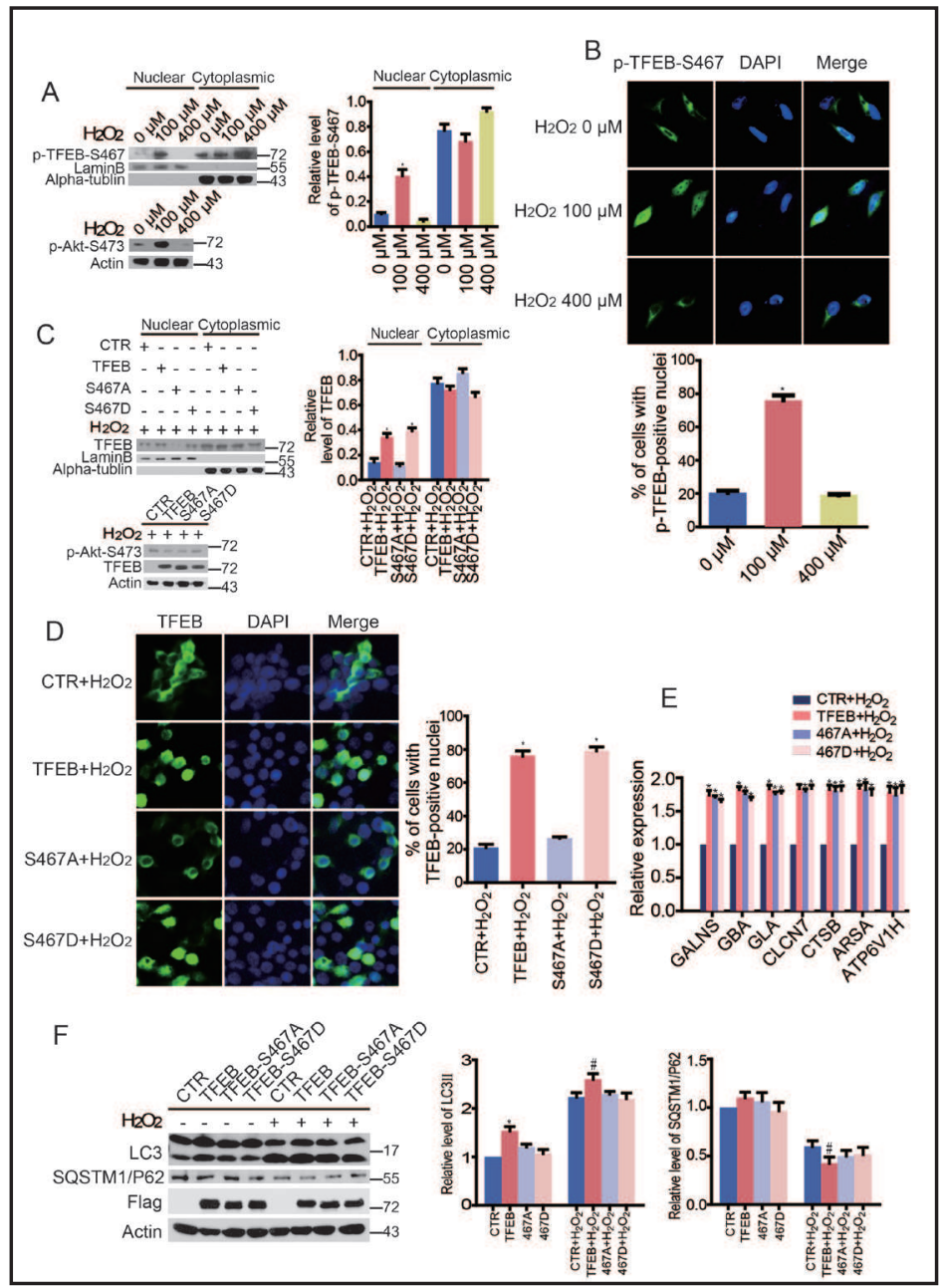
with $\mathrm{H}_{2} \mathrm{O}_{2}(400 \mu \mathrm{M})$ for $24 \mathrm{~h}$. The subcellular fractions were separated, TFEB in the cytoplasm and nucleus was analyzed by Western blotting, whereas band intensities normalized to lamin B or $\alpha$-tublin are shown on the right, values are the mean \pm SEM of 3 independent experiments. $* P<0.05$ versus Flag-vector (CTR) or TFEB-S467 A $+\mathrm{H}_{2} \mathrm{O}_{2}$ group (C). The localization of TFEB was detected by immunofluorescence staining and observed via confocal microscopy. The percentage of TFEB-positive nuclei in SH-SY5Y cells is shown on the right, values are the mean \pm SEM of 3 independent experiments in which 100 cells were analyzed. $* P<0.05$ versus Flag-vector (CTR) or TFEB$\mathrm{S} 467 \mathrm{~A}+\mathrm{H}_{2} \mathrm{O}_{2}$ group (D). The relative expression of lysosome genes were analyzed by RT-PCR, and gene expression was normalized relative to the housekeeping gene, GAPDH. Values are the mean \pm SEM of 3 independent experiments. $* P<0.05$ versus Flag-vector (CTR) $+\mathrm{H}_{2} \mathrm{O}_{2}$ group (E). (F) SH-SY5Y cells were infected with lentiviruses Flag-vector (CTR), Flag-TFEB, Flag-TFEB-S467A or Flag-TFEB-S467D for $48 \mathrm{~h}$, and then treated with MPTP ( $400 \mu \mathrm{M})$ for $24 \mathrm{~h}$. LC3, and SQSTM1/P62 were analyzed by Western blotting, whereas band intensities normalized to $\beta$-actin are shown on the right, values are the mean \pm SEM of 3 independent experiments. $* P<0.05$ versus CTR group, ${ }^{\#} P<0.05$ versus $\mathrm{CTR}+\mathrm{H}_{2} \mathrm{O}_{2}$ group.

the effects of an Akt activator and inhibitor on TFEB-Ser467 phosphorylation. Coimmunoprecipitation assay showed that the S467A substitution completely abolished TFEB phosphorylation by Akt (Fig. 5E). In further experiments, mutation of Ser467 prevented phosphorylation of TFEB-Ser467 by Akt in response to EGF, but did not affect EGF-induced phosphorylation of Akt-Ser473 (Fig. 5F). Similarly, in the stable cell lines expressing FlagTFEB or TFEB-S467A, the Akt inhibitor suppressed phosphorylation of TFEB-Ser467 and Akt-Ser473 (Fig. 5F). Further, the SH-SY5Y cells were incubated with either Wortmannin, 


\section{Cellular Physiology Cell Physiol Biochem 2018;46:1536-1554 \begin{tabular}{l|l} 
DOI 10.1159/000489198 & O2018 The Author(s) \\
www.karger.com/cpb
\end{tabular} \\ Su et al.: TFEB is Involved in $\mathrm{H}_{2} \mathrm{O}_{2}$-Induced Neuronal Apoptosis}

Akt inhibitor, or PD98059 and then treated with EGF. The results showed that EGF markedly induced TFEB-Ser467 phosphorylation, and inhibiting PI3K/Akt signalling with Wortmannin or FAkt inhibitor dramatically reduced TFEB-Ser467 phosphorylation induced by insulin treatment (Fig. 5G). In addition, the wild-type TFEB and TFEB-S467A mutant purified by Flag pull down were incubated with the Akt-CA; in vitro kinase assay showed that mutant of TFEB-S467 A could not be phosphorylated by Akt (Fig. 5H). Together, these results clearly suggested that TFEB is phosphorylated at Ser467 by Akt.

Phosphorylated TFEB translocates into the nucleus but does not regulate lysosome gene expression under oxidative stress

Because Akt can phosphorylate TFEB at Ser467, we sought to verify whether phosphorylated TFEB was translocated into the nucleus under oxidative stress. Thus, SHSY5Y cells were treated with different concentrations of $\mathrm{H}_{2} \mathrm{O}_{2}$, and a nuclear and cytoplasmic extract was prepared from SH-SY5Y cells. Western blot analysis shows that the level of phosphorylated TFEB at S467 significantly increased in the nuclei of cells treated with 100 $\mu \mathrm{M} \mathrm{H}_{2} \mathrm{O}_{2}$ compared with that in those untreated or treated with $400 \mu \mathrm{M} \mathrm{H}_{2} \mathrm{O}_{2}$, accompanied by increased Akt S473 phosphorylation (Fig. 6A). Notably, in the nucleus treated with 400 $\mu \mathrm{M} \mathrm{H}_{2} \mathrm{O}_{2}$, TFEB S467 phosphorylation was significantly decreased (Fig. 6A). These findings indicate that a low concentration of $\mathrm{H}_{2} \mathrm{O}_{2}$ may induce TFEB S467 phosphorylation and nuclear translocation, whereas a high concentration of $\mathrm{H}_{2} \mathrm{O}_{2}$ reduces TFEB phosphorylation at S467 and nuclear translocation. To provide additional confirmation that phosphorylated TFEB can translocate into the nucleus, we performed immunofluorescence staining for TFEB-S467 in SH-SY5Y cells. The results show that treating the cells with $100 \mu \mathrm{M} \mathrm{H}_{2} \mathrm{O}_{2}$ but not $400 \mu \mathrm{M} \mathrm{H}_{2} \mathrm{O}_{2}$ facilitated TFEB translocation into the nucleus (Fig. 6B). Further, the SHSY5Y cells were infected with lentivirus encoding CTR/TFEB/TFEB-S467A/D and treated with $400 \mathrm{\mu M} \mathrm{H}_{2} \mathrm{O}_{2}$. As shown in Fig. 6C, compared with CTR or TFEB-S467A, TFEB and TFEBS467D were translocated into the nucleus by more than 2-fold in response to $400 \mu \mathrm{M} \mathrm{H}_{2} \mathrm{O}_{2}$. Immunofluorescence staining further confirmed that the percentages of TFEB-positive nuclei were significantly augmented in the cells transfected with TFEB and TFEB-S467D compared with those in cells transfected with CTR or TFEB-S467A (Fig. 6D). These results suggested that phospho-mimic TFEB-S467D mutant but not a non-phosphorylated TFEBS467A mutant was more easily translocated into the nucleus in response to oxidative stress.

A recent study indicates that TFEB could translocate into the nucleus and induce autophagy through the lysosomal pathway after exposure of SH-SY5Y cells to $100 \mu \mathrm{M}$ $\mathrm{H}_{2} \mathrm{O}_{2}$ [35]. Therefore, we wanted to confirm whether pTFEB-Ser467 translocates into the nucleus to regulate lysosome or autophagy genes. The expression levels of lysosome genes were determined in TFEB-, TFEB-S467A- or TFEB-S467D-overexpressing SH-SY5Y cells treated with $\mathrm{H}_{2} \mathrm{O}_{2}$. The results indicated that although expression of lysosome genes was upregulated in TFEB-, TFEB-S467A- or TFEB-S467D-transduced cells (Fig. 6E), there is no significant difference between TFEB-S467A- and TFEB-S467D-transduced cells (Fig. 6E). To further determine whether autophagy genes were affected by TFEB-Ser467 phosphorylation, SH-SY5Y cells overexpressing wild-type TFEB, TFEB-S467A, and TFEBS467D were treated with or without $\mathrm{H}_{2} \mathrm{O}_{2}$. Western blot analysis of LC3 II and SQSTM1/ P62 showed that the overexpression of TFEB increased LC3 II expression and decreased SQSTM1/P62 expression (Fig. 6F). The expression of LC3 II and SQSTM1/P62 revealed no significant differences between TFEB-S467A- and TFEB-S467D-transduced cells (Fig. 6F), suggesting that phosphorylation of TFEB at Ser467 by Akt does not affect the expression of lysosome or autophagy genes under oxidative stress.

\section{The phosphorylation of TFEB at Ser467 by Akt inhibits SH-SY5Y cell apoptosis}

Next, we investigated the effect of TFEB-Ser467 phosphorylation on SH-SY5Y cell apoptosis. SH-SY5Y cells were infected with lentivirus encoding wild-type TFEB, TFEBS467A, and TFEB-S467D, followed by incubation without or with $\mathrm{H}_{2} \mathrm{O}_{2}$. Western blot analysis showed that the overexpression of TFEB and TFEB-S467D but not TFEB-S467A markedly 


\section{Cellular Physiology Cell Physiol Biochem 2018;46:1536-1554 \begin{tabular}{ll|l} 
DOI: 10.1159/000489198 & O 2018 The Author(s). Published by S. Karger AG, Basel \\
www.karger.com/cpb
\end{tabular}

Fig. 7. The phosphorylation of TFEB at Ser467 by Akt suppresses SH-SY5Y cell apoptosis. (A) SH-SY5Y cells were infected with lentiviruses Flag-vector (CTR), Flag-TFEB, Flag-TFEB-S467A, or FlagTFEB-S467D for $48 \mathrm{~h}$, and then treated with or without $\mathrm{H}_{2} \mathrm{O}_{2}(400 \mu \mathrm{M})$ for 24 h. PARP cleavage and Akt phosphorylation were analyzed by Western blotting, whereas band intensities normalized to $\beta$-actin are shown on the right, values are the mean \pm SEM of 3 independent experiments. $* P<0.05$ versus corresponding CTR group, ${ }^{\#} P<0.05$ versus $467 \mathrm{D}$ group. (B, C) SH-SY5Y cells were infected with the indicated recombinant lentiviruses for $48 \mathrm{~h}$, and pretreated with Akt inhibitor for $1 \mathrm{~h}$, or with EGF for $15 \mathrm{~min}$, and then treated with $\mathrm{H}_{2} \mathrm{O}_{2}(400 \mu \mathrm{M})$ for $24 \mathrm{~h}$. PARP cleavage and Akt phosphorylation were analyzed by Western blotting, whereas band intensities normalized to $\beta$-actin are shown below the Western blotting, values are the mean \pm SEM of 3 independent experiments. $* P<0.05$ versus corresponding CTR group, ${ }^{*} P<0.05$ versus $467 \mathrm{D}+\mathrm{H}_{2} \mathrm{O}_{2}$ group. (D) SH-SY5Y cells were infected and treated as described in (B, C). Cell apoptosis was detected by flow cytometry. The $x$-axis represents the fluorescence intensity of Annexin-V-PE, and the y-axis represents the fluorescence intensity of 7-AAD. The data of flow cytometry were quantified are shown on the right. Values are the mean \pm SEM. $* P<0.05$ versus their corresponding control group, ${ }^{\#} P<0.05$ versus S467D $+\mathrm{H}_{2} \mathrm{O}_{2}$ group. (E, F) Primary cultures of mouse neurons were infected and treated as described above. PARP cleavage and Akt phosphorylation were analyzed by Western blotting, whereas band intensities normalized to $\beta$-actin are shown on the right, values are the mean \pm SEM of 3 independent experiments. $* P<0.05$ versus their corresponding control group (E). Cell apoptosis was detected by flow cytometry. The $x$-axis represents the fluorescence intensity of Annexin-V-PE, and the y-axis represents the fluorescence intensity of 7-AAD. The data of flow cytometry were quantified are shown on the right. Values are the mean \pm SEM. $* P<0.05$ versus their corresponding control group, ${ }^{\#} P<0.05$ versus $\mathrm{S} 467 \mathrm{D}+\mathrm{H}_{2} \mathrm{O}_{2}$ group (F).

decreased the cleavage of PARP induced by $\mathrm{H}_{2} \mathrm{O}_{2}$ (Fig. 7A, lanes 6 and 8 versus lane 7). To further determine whether apoptosis suppressed by TFEB-Ser467 phosphorylation depends
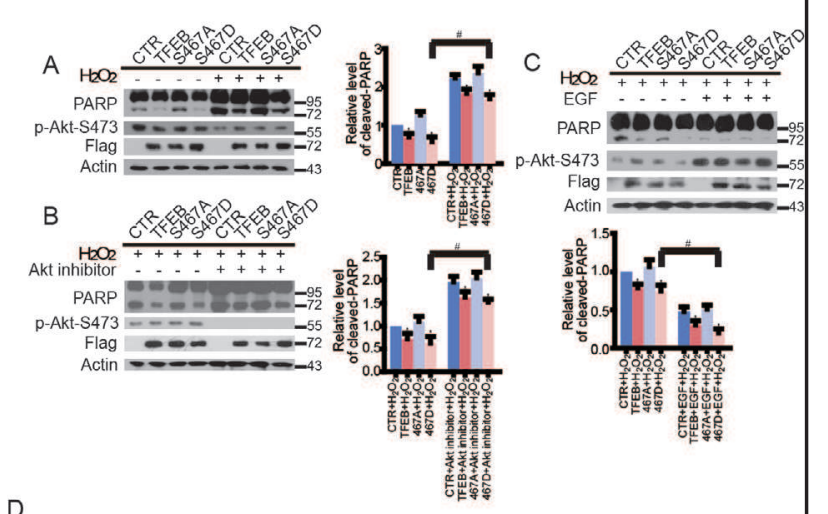

D
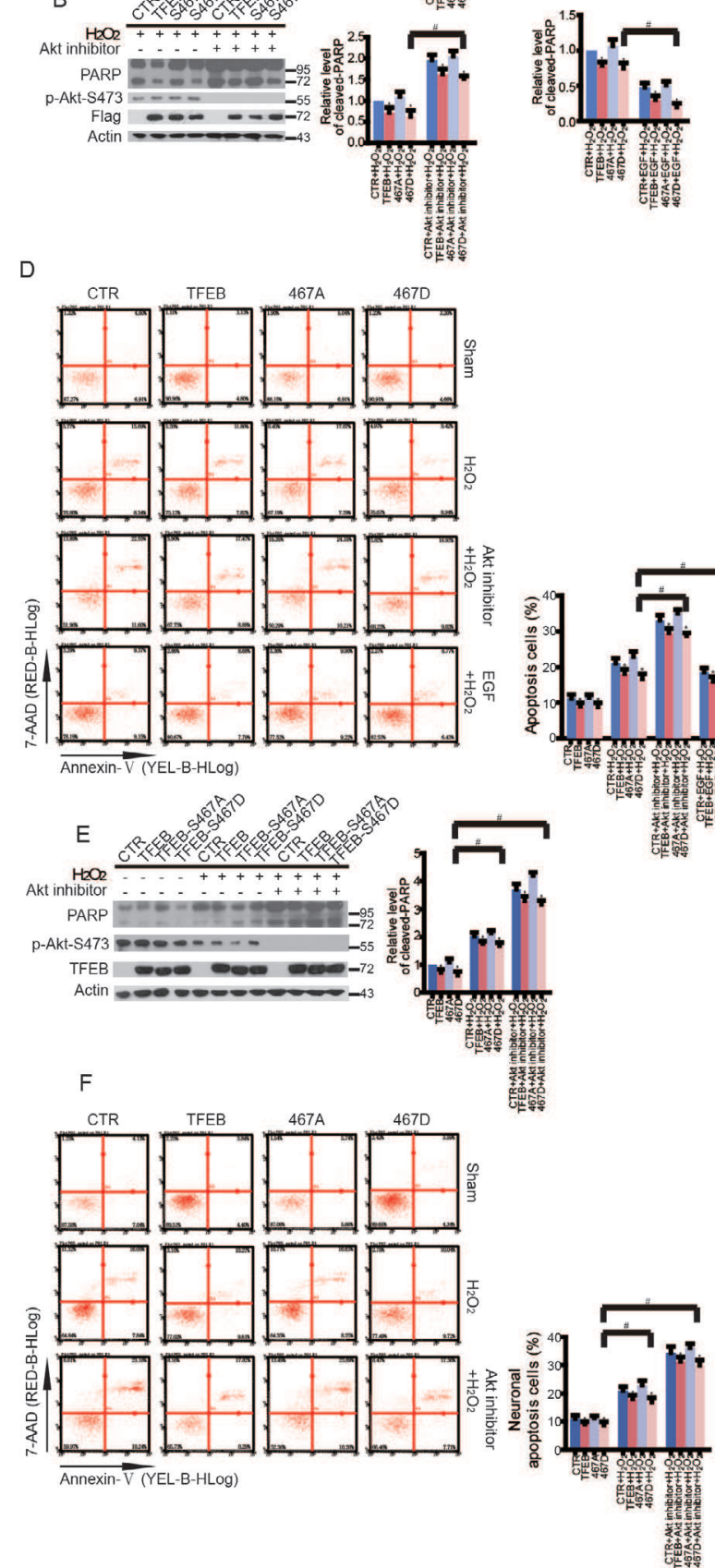

Actin -4

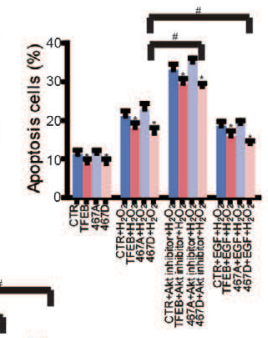




\section{Cellular Physiology Cell Physiol Biochem 2018;46:1536-1554 and Biochemistry Published online: April 24, $2018 \quad$\begin{tabular}{l|l} 
O 2018 The Author \\
www.karger.com/cpp
\end{tabular} \\ Su et al.: TFEB is Involved in $\mathrm{H}_{2} \mathrm{O}_{2}$-Induced Neuronal Apoptosis}

on Akt signalling, we pretreated SH-SY5Y cells overexpressing wild-type TFEB, TFEB-S467 A, and TFEB-S467D with an Akt inhibitor or EGF prior to $\mathrm{H}_{2} \mathrm{O}_{2}$ treatment. Western blot analysis of cleaved PARP showed that compared with overexpression of TFEB-S467A, overexpression of TFEB-S467D decreased the cleavage of PARP induced by $\mathrm{H}_{2} \mathrm{O}_{2}$ alone or together with the Akt inhibitor (Fig. 7B, lanes 4 and 8 versus lanes 3 and 7). However, activating Akt with EGF in TFEB-S467A-overexpressing cells reduced $\mathrm{H}_{2} \mathrm{O}_{2}$-induced PARP cleavage to a lesser extent than that in wild-type TFEB- or TFEB-S467D-overexpressing cells (Fig. 7C).

Flow cytometry data revealed that the overexpression of both TFEB and TFEB-S467D significantly suppressed cell apoptosis regardless of $\mathrm{H}_{2} \mathrm{O}_{2}$ treatment, and S467A point mutation abrogated the inhibitory effect on $\mathrm{H}_{2} \mathrm{O}_{2}$-induced cell apoptosis (Fig. 7D). In further experiments, TFEB-S467D overexpression also reduced SH-SY5Y cell apoptosis induced by $\mathrm{H}_{2} \mathrm{O}_{2}$ alone or together with an Akt inhibitor. Activating Akt with EGF could not prevent $\mathrm{H}_{2} \mathrm{O}_{2}-$ induced apoptosis in TFEB-S467A-overexpressing SH-SY5Y cells (Fig. 7D).

We additionally studied the effect of TFEB-Ser467 phosphorylation on primary neuronal apoptosis. Primary cultures of mouse neurons were infected with adenovirus encoding wild-type TFEB, TFEB-S467A, and TFEB-S467D and were treated with an Akt inhibitor prior to $\mathrm{H}_{2} \mathrm{O}_{2}$ treatment. Western blot analysis of cleaved PARP showed that compared with overexpression of TFEB-S467A, overexpression of TFEB-S467D decreased the cleavage of PARP induced by $\mathrm{H}_{2} \mathrm{O}_{2}$ alone or together with the Akt inhibitor (Fig. 7E, lanes 8 and 12 versus lanes 7 and 11). Consistent with the Western blot results, flow cytometry data revealed that TFEB-S467D overexpression significantly reduced primary neuronal apoptosis induced by $\mathrm{H}_{2} \mathrm{O}_{2}$ alone or together with the Akt inhibitor (Fig. 7F). These findings suggested that the phosphorylation of TFEB at Ser467 by Akt is necessary for inhibition of $\mathrm{H}_{2} \mathrm{O}_{2}$-induced neuronal apoptosis.

Phosphorylation of TFEB at Ser467 protects dopaminergic neurons under MPTP-induced oxidative stress

MPTP, which has been reported to kill dopaminergic neurons to induce parkinsonism through inhibition of mitochondrial complex I, has also been shown to be associated with the production of $\mathrm{O}_{2}^{-}$radicals in vitro $[7,41]$. Thus, we investigated whether phosphorylation of TFEB at Ser467 could protect dopaminergic neurons in the whole animal. We overexpressed wild-type TFEB, TFEB-S467A, and TFEB-S467D in mouse midbrain by injecting the recombinant adenoviruses and observed the effect of their overexpression on MPTP-induced neuronal apoptosis. As shown in Fig. 8A, exogenous GFP, TFEB, TFEB-S467A, and TFEBS467D could be expressed in midbrain dopaminergic neurons of mice. Immunostaining of tyrosine hydroxylase revealed that TFEB and TFEB-S467D overexpression significantly increased TH-positive neurons on the injected sides compared with those on the uninjected sides (Fig. 8B). These findings clearly suggested that phosphorylation of TFEB at Ser467 can effectively protect dopaminergic neurons in the whole animal under MPTP-induced oxidative stress.

\section{Discussion}

In this study, we demonstrated that the phosphorylation of TFEB by Akt can suppress SH-SY5Y cell apoptosis induced by oxidative stress. The oxidative stress-induced apoptosis is aggravated when PI3K/Akt signaling is specifically blocked by their corresponding inhibitor. Reversely, Akt signaling activation by EGF facilitates cell survival under oxidative stress. Moreover, TFEB overexpression decreases $\mathrm{H}_{2} \mathrm{O}_{2}$-induced apoptosis in EGF-treated cells. These findings suggested that Akt-promoted neuronal survival is mediated by TFEB. We further found that Akt physically interacts with TFEB through the HLH domain of TFEB and Akt-tail. Our results showed that mutation of Ser467 can prevent the phosphorylation of TFEB by Akt in response to EGF, and loses its inhibitory effect on $\mathrm{H}_{2} \mathrm{O}_{2}$-induced cell apoptosis. The phospho-mimic TFEB-S467D mutant, but not a non-phosphorylated TFEB- 


\section{Cellular Physiology \begin{tabular}{ll|l} 
and Biochemistry & $\begin{array}{l}\text { DOI: 10.1159/000489198 } \\
\text { Published online: Apri 24, } 2018\end{array}$ & $\begin{array}{l}\text { (c) } 2018 \text { The Author(s). Published by S. Karger AG, Basel } \\
\text { www.karger.com/cpb }\end{array}$ \\
\hline
\end{tabular} \\ Su et al.: TFEB is Involved in $\mathrm{H}_{2} \mathrm{O}_{2}$-Induced Neuronal Apoptosis}

Fig. 8. Phosphorylation of TFEB at Ser467 protects dopaminergic neurons under MPTP-induced oxidative stress. (A) Adenovirus vector encoding GFP, TFEB, TFEBS467A, or TFEB-S467D was stereotaxically injected into mouse midbrain ( $\mathrm{n}=6$ mice per group). Dopaminergic neurons were detected via immunohistochemical staining using tyrosine hydroxylase (TH) antibodies, and the viral expression of GFP was shown by the fluorescence intensity of GFP. Cells were stained for GFP (green), tyrosine hydroxylase (red), or their co-localization (yellow). (B) Four recombinant adenoviruses as described in (A) were injected into mouse midbrain prior to injection of MPTP. Dopaminergic neurons were immunostained by using $\mathrm{TH}$ antibodies. The numbers of $\mathrm{TH}-$ positive neurons were quantified are shown on the right. Values are the mean \pm SEM. $* P<0.05$ versus GFP or S467A group.

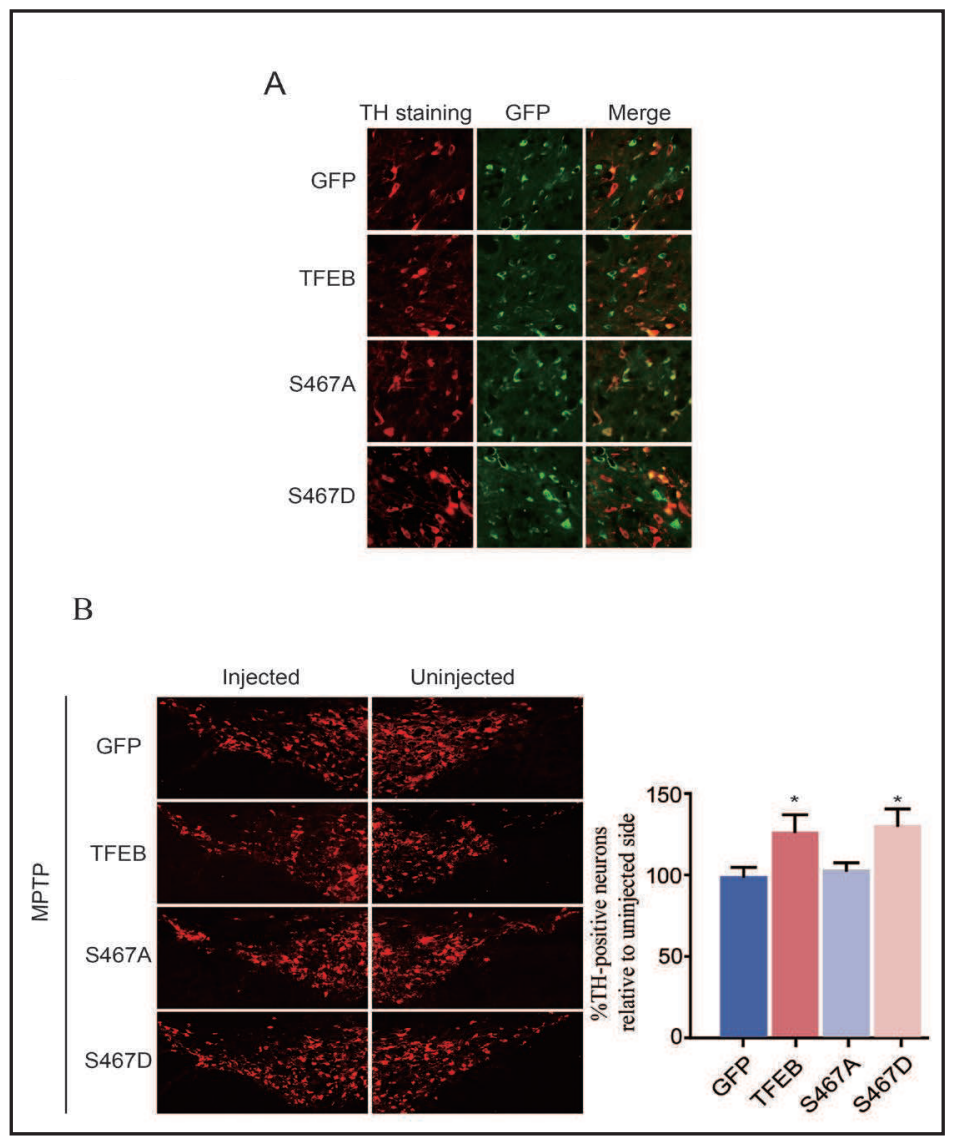

S467A mutant can suppress can suppress $\mathrm{H}_{2} \mathrm{O}_{2}$-induced cell apoptosis. In the whole animal, we also found that TFEB and TFEB-S467D overexpression mediated by adenovirus can effectively protect dopaminergic neurons under MPTP-induced oxidative stress. Overall, our results indicate that phosphorylation of TFEB at Ser467 by Akt is necessary for inhibition of oxidative stress-induced neuronal apoptosis in vitro and in vivo.

Akt/protein kinase B is a serine/threonine kinase that promotes cell survival, growth and proliferation and suppresses cell apoptosis $[14,20]$. The pro-survival function of Akt/ protein kinase $\mathrm{B}$ depends on its phosphorylation of a number of downstream targets to inhibit cell death. Multiple pro-apoptosis proteins, including Bad [42], Bax [43], caspase-9 [44], glycogen synthase kinase-3- $\beta$ (GSK-3 $\beta$ ) [45], and forkhead transcription factors [46], are also phosphorylated by Akt. Akt not only phosphorylates these pro-apoptotic proteins, but also inactivates them, and retains them in the cytoplasm to inhibit the transcription of relevant pro-apoptotic genes, thereby suppressing cell apoptosis. In addition, Akt can also activate the transcription factor NF- $\mathrm{BB}$ to upregulate its DNA-binding activity and exert prosurvival functions [47]. In this study, the transcription factor TFEB is a downstream target of Akt. TFEB association with Akt was mediated by the HLH domain of TFEB and Akt-tail, and TFEB was phosphorylated at Ser467 by Akt signaling, which in turn suppresses cell apoptosis induced by $\mathrm{H}_{2} \mathrm{O}_{2}$ in neuronal cells. The insight into the mechanism of anti-apoptotic effect of TFEB might provide novel therapeutic strategies for neurodegenerative diseases.

Neuronal cells in the brain are highly sensitive to oxidative stress; therefore, excessive ROS generation can cause damage of neuronal cells via either an apoptotic or a necrotic pathway [48]. Oxidative stress is also a major cause for PD. Recent evidence has suggested an intimate link between oxidative stress-induced neuronal apoptosis and autophagy, which is an essential cellular antioxidant pathway in neurodegenerative disease $[49,50]$. And TFEB could upregulate antophagy-lysosome gene expression and lysosome activity [35]. Recent evidence has suggested sublethal oxidative stress $\left(50 \mu \mathrm{M} \mathrm{H}_{2} \mathrm{O}_{2}\right)$ activates an mTOR- 


\section{Cellular Physiology Cell Physiol Biochem 2018;46:1536-1554 \begin{tabular}{l|l} 
DOI 10.1159/000489198 & 2018 The Author(s) \\
www.karger.com/cpb
\end{tabular} \\ Su et al.: TFEB is Involved in $\mathrm{H}_{2} \mathrm{O}_{2}$-Induced Neuronal Apoptosis}

independent signaling pathway involving the activation of LMP-cathepsin-caspase 3 axis leading to the transcription of TFEB-target genes involved in lysosome biogenesis [51]. In the present study, we demonstrated that TFEB was phosphorylated by Akt and mediated the pro-survival function of Akt in EGF-treated SH-SY5Y cells, and the phosphorylated TFEB inhibited neuronal apoptosis. Importantly, we found that the phospho-mimic TFEB-S467D mutant, but not a non-phosphorylated TFEB-S467A mutantcan markedly inhibit $\mathrm{H}_{2} \mathrm{O}_{2}$ induced cell apoptosis via translocating into the nucleus under oxidative stress. However, the expression of lysosome and autophagy genes revealed no significant differences between TFEB-S467A- and TFEB-S467D-transduced cells. Thus, we hypothesized that Akt-dependent phosphorylation of TFEB in neuronal cells plays an essential role in oxidative stress-induced apoptosis, possibly independent of TFEB-regulated autophagy gene expression. Our findings indicated that low concentration of $\mathrm{H}_{2} \mathrm{O}_{2}(100 \mu \mathrm{M}$ and $200 \mu \mathrm{M})$ facilitates neuronal survival by inducing Akt S473 and TFEB S467 phosphorylation, whereas high concentration of $\mathrm{H}_{2} \mathrm{O}_{2}$ $(400 \mu \mathrm{M}$ and $800 \mu \mathrm{M})$ promotes SH-SY5Y cell apoptosis via suppressing Akt S473 and TFEB S467 phosphorylation. These results suggest that the effects of $\mathrm{H}_{2} \mathrm{O}_{2}$ on neuronal cell fate depend on its concentration used.

TFEB is known to regulate lysosomal biogenesis and autophagy [23-25] and plays an important role in cell survival under oxidative stress [35]. The subcellular localization and activity of TFEB are regulated by its phosphorylation status [52]. Previous studies have indicated that increasing reactive oxygen species (ROS) levels directly activates MCOLN1 to induce lysosome $\mathrm{Ca}^{2+}$ release, triggering PPP3/calcineurin-dependent TFEB nuclear trnslocation to enhance autophagy [53]. A recent study indicates that TFEB could translocate into the nucleus and induced autophagy by lysosomal pathway after exposure of SH-SY5Y cells to $100 \mu \mathrm{M} \mathrm{H}_{2} \mathrm{O}_{2}$, and that the C-ETS2-TFEB axis promoted neuron survival under oxidative stress by regulating lysosome activity [35]. Therefore, we wanted to confirm whether the phosphorylation of TFEB suppressed SH-SY5Y cell apoptosis by regulating lysosome or autophagy genes. The mRNA expression levels of lysosome genes and protein expression of autophagy genes were determined in TFEB-S467A- and TFEB-S467D-overexpressing SH-SY5Y cells treated with $\mathrm{H}_{2} \mathrm{O}_{2}$. The results indicated that the expression of lysosome and autophagy genes revealed no significant differences between TFEB-S467A- and TFEBS467D-transduced cells, suggesting that phosphorylation of TFEB at Ser467 by Akt does not mediate lysosome or autophagy genes to suppress cell apoptosis under oxidative stress.

Previous studies have indicated that TFEB can be phosphorylated by rapamycin complex 1 (mTORC1) at Ser211 [54], as well as by extracellular signal-regulated kinase 2 (ERK2) at Ser142 [23]. TFEB phosphorylated by mTORC1 and ERK2 is retained in the cytosol, and the activity is inhibited by its serine phosphorylation. But when TFEB-Ser211 or TFEB-Ser142 was mutated, TFEB translocated to the nucleus and kept active to regulate downstream genes [23, 54-56]. It has been demonstrated that a key effector of Akt is the mammalian target of rapamycin (mTOR). Akt can stimulate mTORC1 activity through phosphorylation of tuberous sclerosis compex 2 (TSC2) and PRAS40, both negative regulators of mTOR activity [57], and in turn, mTORC1 phosphorylates TFEB, the phosphorylated TFEB by mTORC1 kept inactive in the cytosol [54-56].

Recent report shows that Akt phosphorylates TFEB atSer467 and represses TFEB nuclear translocation independently of mechanistic target of rapamycin complex 1 (mTORC1). The autophagy enhancer trehalose activates TFEB by diminishing Akt activity [38], and Akt pharmacological inhibition promotes cellular clearance in a variety of models of genetic diseases presenting with impairment of lysosomal pathways [58]. In the present study, our data indicate that Akt could physically interact with TFEB through its Akt-tail and the HLH domain of TFEB, and directly phosphorylated TFEB at Ser467, and the phosphorylated TFEB by Akt in SH-SY5Y cells suppressed cell apoptosis under oxidative stress. Moreover, Akt inhibitors increased oxidative stress-induced apoptosis but did not activate autophagy/ lysosomal pathway in our model. There is a discrepancy between the findings of the present study and that of previously published work [38]. We speculate that these discrepancies could be attributable to different cell lines used by two different groups. For example, 


\section{Cellular Physiology Cell Physiol Biochem 2018;46:1536-1554 \begin{tabular}{c|c|c|c|}
\cline { 2 - 3 } & DOI: 10.1159/000489198 & (C) 2018 The Author(s). Published by S. Karger AG, Base
\end{tabular} and Biochemistry Published online: April 24, 2018 www.karger.com/cpb \\ Su et al.: TFEB is Involved in $\mathrm{H}_{2} \mathrm{O}_{2}$-Induced Neuronal Apoptosis}

Palmieri et al. used autophagy enhancer trehalose to treat HeLa or cultured cortical neurons from mice [38], whereas we used different concentrations of $\mathrm{H}_{2} \mathrm{O}_{2}$ to treat human neuroblastoma SH-SY5Y cells or HEK293T cells stablely expressing Flag-TFEB. Although we did not explore how phosphorylated TFEB regulates its target gene transcription, we think that TFEB translocated into the nucleus exerts a protective effect against oxidative stress via regulating expression of apoptosis- or survival-related genes. Akt can induce survival/ protection against oxidative stress by a number of mechanisms, activation of TFEB by Akt signaling may be one of mechanisms whereby Akt exerts a protective effect against oxidative stress.

However, our findings were mainly obtained in TFEB-S467A- or TFEB-S467Doverexpressing SH-SY5Y cells. It is well known that Ser to Ala mutation always abolishes phosphorylation, while Ser to Asp mutation does not necessarily mimic constitutive phosphorylation but has other effects. In this study, we found that TFEB-S467D mutant was more easily translocated into the nucleus than non-phosphorylated TFEB-S467A mutant under oxidative stress, implying that TFEB-S467D might act as a "phosphomimetic" to some extent. In our next investigation, we plan to explore whether TFEB-S467D may activate downstream target genes.

\section{Conclusion}

In summary, our data support the following conclusions: i) low concentration of $\mathrm{H}_{2} \mathrm{O}_{2}$ induces TFEB phosphorylation at Ser467 and nuclear translocation by phosphorylating Akt, facilitating neuronal survival; ii) high concentration of $\mathrm{H}_{2} \mathrm{O}_{2}$ promotes SH-SY5Y cell apoptosis via suppressing TFEB S467 phosphorylation and nuclear translocation, iii) TFEBS467D mutant was more easily translocated into the nucleus than non-phosphorylated TFEB-S467A mutant under oxidative stress. Thus, TFEB-S467D, as a "phosphomimetic", might be used to inhibit cell apoptosis induced by high concentration of $\mathrm{H}_{2} \mathrm{O}_{2}$, providing a novel therapeutic strategy for neurodegenerative diseases.

\section{Acknowledgements}

This study was supported by the National Natural Science Foundation of China (No. 31671182).

\section{Disclosure Statement}

The authors declare there are no conflicts of interest.

\section{References}

1 Gilgun-Sherki Y, Rosenbaum Z, Melamed E, Offen D: Antioxidant therapy in acute central nervous system injury: current state. Pharmacol Rev 2002;54:271-284.

-2 Slemmer JE, Zhu C, Landshamer S, Trabold R, Grohm J, Ardeshiri A, Wagner E, Sweeney MI, Blomgren K, Culmsee C, Weber JT, Plesnila N: Causal role of apoptosis-inducing factor for neuronal cell death following traumatic brain injury. Am J Pathol 2008;173:1795-1805.

-3 Herrmann AM, Gobel K, Simon OJ, Melzer N, Schuhmann MK, Stenner MP, Weishaupt A, Kleinschnitz C, Bittner S, Meuth P, Stuve O, Budde T, Kieseier BC, Wiendl H, Meuth SG: Glatiramer acetate attenuates proinflammatory $\mathrm{T}$ cell responses but does not directly protect neurons from inflammatory cell death. Am J Pathol 2010;177:3051-3060.

4 Suzuki J, Iwai M, Mogi M, Oshita A, Yoshii T, Higaki J, Horiuchi M: Eplerenone with valsartan effectively reduces atherosclerotic lesion by attenuation of oxidative stress and inflammation. Arterioscler Thromb Vasc Biol 2006;26:917-921.

5 Blesa J, Trigo-Damas I, Quiroga-Varela A, Jackson-Lewis VR: Oxidative stress and Parkinson's disease. Front Neuroanat 2015;9:91.

-6 Tsang AH, Chung KK: Oxidative and nitrosative stress in Parkinson's disease. Biochim Biophys Acta 2009;1792:643-650. 


\section{Cellular Physiology Cell Physiol Biochem 2018;46:1536-1554 \begin{tabular}{c|l|l} 
DOI: 10.1159/000489198 & $\begin{array}{l}\text { () 2018 The Author(s). Published by S. Karger AG, Basel } \\
\text { www.karger.com/cpb }\end{array}$
\end{tabular}}

Su et al.: TFEB is Involved in $\mathrm{H}_{2} \mathrm{O}_{2}$-Induced Neuronal Apoptosis

7 Zhou C, Huang Y, Przedborski S: Oxidative stress in Parkinson's disease: a mechanism of pathogenic and therapeutic significance. Ann N Y Acad Sci 2008;1147:93-104.

8 Korsager Larsen M, Matchkov VV: Hypertension and physical exercise: The role of oxidative stress. Medicina (Kaunas) 2016;52:19-27.

\9 Mohamed J, Nazratun Nafizah AH, Zariyantey AH, Budin SB: Mechanisms of Diabetes-Induced Liver Damage: The role of oxidative stress and inflammation. Sultan Qaboos Univ Med J 2016;16:e132-141.

10 Lo EH, Dalkara T, Moskowitz MA: Mechanisms, challenges and opportunities in stroke. Nat Rev Neurosci 2003;4:399-415.

11 Olanow CW: The pathogenesis of cell death in Parkinson's disease--2007 Mov Disord 2007;22 Suppl 17:S335-342.

12 Vila M, Przedborski S: Targeting programmed cell death in neurodegenerative diseases. Nat Rev Neurosci 2003;4:365-375.

13 Mattson MP: Apoptosis in neurodegenerative disorders. Nat Rev Mol Cell Biol 2000;1:120-129.

14 Hemmings BA, Restuccia DF: PI3K-PKB/Akt pathway. Cold Spring Harb Perspect Biol 2012;4:a011189.

15 Dudek H, Datta SR, Franke TF, Birnbaum MJ, Yao R, Cooper GM, Segal RA, Kaplan DR, Greenberg ME: Regulation of neuronal survival by the serine-threonine protein kinase Akt. Science 1997;275:661-665.

-16 Jha SK, Jha NK, Kar R, Ambasta RK, Kumar P: p38 MAPK and PI3K/AKT Signalling Cascades inParkinson's Disease. Int J Mol Cell Med 2015;4:67-86.

17 Wang G, Pan J, Chen SD: Kinases and kinase signaling pathways: potential therapeutic targets in Parkinson's disease. Prog Neurobiol 2012;98:207-221.

18 Kim SR, Chen X, Oo TF, Kareva T, Yarygina O, Wang C, During M, Kholodilov N, Burke RE: Dopaminergic pathway reconstruction by Akt/Rheb-induced axon regeneration. Ann Neurol 2011;70:110-120.

19 Timmons S, Coakley MF, Moloney AM, C ON: Akt signal transduction dysfunction in Parkinson's disease. Neurosci Lett 2009;467:30-35.

20 Franke TF, Hornik CP, Segev L, Shostak GA, Sugimoto C: PI3K/Akt and apoptosis: size matters. Oncogene 2003;22:8983-8998.

21 Lin HJ, Wang X, Shaffer KM, Sasaki CY, Ma W: Characterization of H2O2-induced acute apoptosis in cultured neural stem/progenitor cells. FEBS Lett 2004;570:102-106.

-22 Jantas D, Piotrowski M, Lason W: An Involvement of PI3-K/Akt Activation and Inhibition of AIF Translocation in Neuroprotective Effects of Undecylenic Acid (UDA) Against Pro-Apoptotic Factors-Induced Cell Death in Human Neuroblastoma SH-SY5Y Cells. J Cell Biochem 2015;116:2882-2895.

-23 Settembre C, Di Malta C, Polito VA, Garcia Arencibia M, Vetrini F, Erdin S, Erdin SU, Huynh T, Medina D, Colella P, Sardiello M, Rubinsztein DC, Ballabio A: TFEB links autophagy to lysosomal biogenesis. Science 2011;332:1429-1433.

24 Settembre C, Ballabio A: TFEB regulates autophagy: an integrated coordination of cellular degradation and recycling processes. Autophagy 2011;7:1379-1381.

25 Sardiello M, Palmieri M, di Ronza A, Medina DL, Valenza M, Gennarino VA, Di Malta C, Donaudy F, Embrione V, Polishchuk RS, Banfi S, Parenti G, Cattaneo E, Ballabio A: A gene network regulating lysosomal biogenesis and function. Science 2009;325:473-477.

26 Martini-Stoica H, Xu Y, Ballabio A, Zheng H: The Autophagy-Lysosomal Pathway in Neurodegeneration: A TFEB Perspective. Trends Neurosci 2016;39:221-234.

27 Klein K, Werner K, Teske C, Schenk M, Giese T, Weitz J, Welsch T: Role of TFEB-driven autophagy regulation in pancreatic cancer treatment. Int J Oncol 2016;49:164-172.

-28 Giatromanolaki A, Kalamida D, Sivridis E, Karagounis IV, Gatter KC, Harris AL, Koukourakis MI: Increased expression of transcription factor EB (TFEB) is associated with autophagy, migratory phenotype and poor prognosis in non-small cell lung cancer. Lung Cancer 2015;90:98-105.

29 Argani P: MiT family translocation renal cell carcinoma. Semin Diagn Pathol 2015;32:103-113.

-30 Parenti G, Andria G, Ballabio A: Lysosomal storage diseases: from pathophysiology to therapy. Annu Rev Med 2015;66:471-486.

31 Pi H, Li M, Tian L, Yang Z, Yu Z, Zhou Z: Enhancing lysosomal biogenesis and autophagic flux by activating the transcription factor EB protects against cadmium-induced neurotoxicity. Sci Rep 2017;7:43466.

- 32 Pena KA, Kiselyov K: Transition metals activate TFEB in overexpressing cells. Biochem J 2015;470:65-76.

-33 Zhang YD, Zhao JJ: TFEB Participates in the Abeta-Induced Pathogenesis of Alzheimer's Disease by Regulating the Autophagy-Lysosome Pathway. DNA Cell Biol 2015;34:661-668.

-34 Tsunemi T, Ashe TD, Morrison BE, Soriano KR, Au J, Roque RA, Lazarowski ER, Damian VA, Masliah E, La Spada AR: PGC-1alpha rescues Huntington's disease proteotoxicity by preventing oxidative stress and promoting TFEB function. Sci Transl Med 2012;4:142ra197.

35 Ma S, Fang Z, Luo W, Yang Y, Wang C, Zhang Q, Wang H, Chen H, Chan CB, Liu Z: The C-ETS2-TFEB Axis Promotes Neuron Survival under Oxidative Stress by Regulating Lysosome Activity. Oxid Med Cell Longev 


\section{Cellular Physiology Cell Physiol Biochem 2018;46:1536-1554 \begin{tabular}{c|c|c|c|c|}
\hline DOI: 10.1159/000489198 & (c) 2018 The Author(s). Published by S. Karger AG, Basel
\end{tabular} and Biochemistry

2016;2016:4693703.

36 Decressac M, Mattsson B, Weikop P, Lundblad M, Jakobsson J, Bjorklund A: TFEB-mediated autophagy rescues midbrain dopamine neurons from alpha-synuclein toxicity. Proc Natl Acad Sci U S A 2013;110:E1817-1826.

37 Kilpatrick K, Zeng Y, Hancock T, Segatori L: Genetic and chemical activation of TFEB mediates clearance of aggregated alpha-synuclein. PLoS One 2015;10:e0120819.

-38 Palmieri M, Pal R, Nelvagal HR, Lotfi P, Stinnett GR, Seymour ML, Chaudhury A, Bajaj L, Bondar VV, Bremner L, Saleem U, Tse DY, Sanagasetti D, Wu SM, Neilson JR, Pereira FA, Pautler RG, Rodney GG, Cooper JD, Sardiello M: mTORC1-independent TFEB activation via Akt inhibition promotes cellular clearance in neurodegenerative storage diseases. Nat Commun 2017;8:14338.

39 Mendez-Armenta M, Nava-Ruiz C, Juarez-Rebollar D, Rodriguez-Martinez E, Gomez PY: Oxidative stress associated with neuronal apoptosis in experimental models of epilepsy. Oxid Med Cell Longev 2014;2014:293689.

40 Nakamura H, Kawakami A, Ida H, Koji T, Eguchi K: EGF activates PI3K-Akt and NF-kappaB via distinct pathways in salivary epithelial cells in Sjogren's syndrome. Rheumatol Int 2007;28:127-136.

41 Hasegawa E, Takeshige K, Oishi T, Murai Y, Minakami S: 1-Methyl-4-phenylpyridinium (MPP+) induces NADH-dependent superoxide formation and enhances NADH-dependent lipid peroxidation in bovine heart submitochondrial particles. Biochem Biophys Res Commun 1990;170:1049-1055.

42 Datta SR, Dudek H, Tao X, Masters S, Fu H, Gotoh Y, Greenberg ME: Akt phosphorylation of BAD couples survival signals to the cell-intrinsic death machinery. Cell 1997;91:231-241.

43 Sadidi M, Lentz SI, Feldman EL: Hydrogen peroxide-induced Akt phosphorylation regulates Bax activation. Biochimie 2009;91:577-585

44 Cardone MH, Roy N, Stennicke HR, Salvesen GS, Franke TF, Stanbridge E, Frisch S, Reed JC: Regulation of cell death protease caspase-9 by phosphorylation. Science 1998;282:1318-1321.

45 Cross DA, Alessi DR, Cohen P, Andjelkovich M, Hemmings BA: Inhibition of glycogen synthase kinase-3 by insulin mediated by protein kinase B. Nature 1995;378:785-789.

46 Brunet A, Bonni A, Zigmond MJ, Lin MZ, Juo P, Hu LS, Anderson MJ, Arden KC, Blenis J, Greenberg ME: Akt promotes cell survival by phosphorylating and inhibiting a Forkhead transcription factor. Cell 1999;96:857-868.

47 Madrid LV, Mayo MW, Reuther JY, Baldwin AS, Jr: Akt stimulates the transactivation potential of the RelA/ p65 Subunit of NF-kappa B through utilization of the Ikappa B kinase and activation of the mitogenactivated protein kinase p38. J Biol Chem 2001;276:18934-18940.

48 Shin EJ, Jeong JH, Chung YH, Kim WK, Ko KH, Bach JH, Hong JS, Yoneda Y, Kim HC: Role of oxidative stress in epileptic seizures. Neurochem Int 2011;59:122-137.

49 Giordano S, Darley-Usmar V, Zhang J: Autophagy as an essential cellular antioxidant pathway in neurodegenerative disease. Redox Biol 2014;2:82-90.

-50 Moors TE, Hoozemans JJ, Ingrassia A, Beccari T, Parnetti L, Chartier-Harlin MC, van de Berg WD: Therapeutic potential of autophagy-enhancing agents in Parkinson's disease. Mol Neurodegener 2017;12:11.

-51 Leow SM, Chua SX, Venkatachalam G, Shen L, Luo L, Clement MV: Sub-lethal oxidative stress induces lysosome biogenesis via a lysosomal membrane permeabilization-cathepsin-caspase 3-transcription factor EB-dependent pathway. Oncotarget 2017;8:16170-16189.

52 Medina DL, Settembre C, Ballabio A: Methods to Monitor and Manipulate TFEB Activity During Autophagy. Methods Enzymol 2017;588:61-78.

53 Zhang X, Yu L, Xu H: Lysosome calcium in ROS regulation of autophagy. Autophagy 2016;12:1954-1955.

54 Martina JA, Chen Y, Gucek M, Puertollano R: MTORC1 functions as a transcriptional regulator of autophagy by preventing nuclear transport of TFEB. Autophagy 2012;8:903-914.

55 Settembre C, Zoncu R, Medina DL, Vetrini F, Erdin S, Erdin S, Huynh T, Ferron M, Karsenty G, Vellard MC, Facchinetti V, Sabatini DM, Ballabio A: A lysosome-to-nucleus signalling mechanism senses and regulates the lysosome via mTOR and TFEB. EMBO J 2012;31:1095-1108.

-56 Roczniak-Ferguson A, Petit CS, Froehlich F, Qian S, Ky J, Angarola B, Walther TC, Ferguson SM: The transcription factor TFEB links mTORC1 signaling to transcriptional control of lysosome homeostasis. Sci Signal 2012;5:ra42.

57 Dan HC, Ebbs A, Pasparakis M, Van Dyke T, Basseres DS, Baldwin AS: Akt-dependent activation of mTORC1 complex involves phosphorylation of mTOR (mammalian target of rapamycin) by IkappaB kinase alpha (IKKalpha). J Biol Chem 2014;289:25227-25240.

-58 Zhang X, Cheng X, Yu L, Yang J, Calvo R, Patnaik S, Hu X, Gao Q Yang M, Lawas M, Delling M, Marugan J, Ferrer M, Xu H: MCOLN1 is a ROS sensor in lysosomes that regulates autophagy. Nat Commun 2016;7:12109. 